\title{
Innate Lymphoid Cells: Regulators of Gut Barrier Function and Immune Homeostasis
}

\author{
Hui Fan, ${ }^{1}$ Aiyun Wang ${ }^{D},{ }^{1}$ Yuan Wang, ${ }^{1}$ Ye Sun, ${ }^{1}$ Jing Han, ${ }^{1}$ Wenxing Chen, ${ }^{1}$ Shijun Wang, \\ Yuanyuan $\mathrm{Wu} \mathbb{D}^{\mathrm{D}}{ }^{1}$ and Yin $\mathrm{Lu} \mathbb{D}^{1}$ \\ ${ }^{1}$ Jiangsu Key Laboratory for Efficacy and Safety Evaluation of Chinese Material Medica, School of Pharmacy, Nanjing University of \\ Chinese Medicine, Nanjing 210023, China \\ ${ }^{2}$ Shandong Co-Innovation Center of TCM Formula, College of Traditional Chinese Medicine, Shandong University of Traditional \\ Chinese Medicine, Shandong 250035, China
}

Correspondence should be addressed to Yuanyuan Wu; ywu@njucm.edu.cn and Yin Lu; luyingreen@njucm.edu.cn

Received 9 June 2019; Accepted 17 September 2019; Published 20 December 2019

Guest Editor: Kong Chen

Copyright ( 2019 Hui Fan et al. This is an open access article distributed under the Creative Commons Attribution License, which permits unrestricted use, distribution, and reproduction in any medium, provided the original work is properly cited.

Innate lymphoid cells (ILCs), identified in the early years of this century as a new class of leukocyte family unlike the B or T lymphocytes, play a unique role bridging the innate and adaptive immune responses in mucosal immunity. Their origin, differentiation, and activation process and functions have caught global interest. Recently, accumulating evidence supports that ILCs are vital regulators for gastrointestinal mucosal homeostasis through interactions with other structural and stromal cells in gut epithelial barriers. This review will explore the functions of ILCs and other cells in maintaining gut homeostasis and feature the crosstalk between ILCs with other cells and potential pharmacotherapy targeting ILCs applicable in intestinal innate immunity.

\section{Introduction}

The gut barrier is a heterogeneous unit composed as a multilayer system and can be simplified as two components: a physical barrier surface and a deep functional barrier. The physical barrier surface prevents bacterial infiltration and adhesion and regulates paracellular diffusion to the host tissues while the deep functional barrier discriminates between pathogens and commensal microorganisms, organizing the immune tolerance and the immune response to pathogens [1]. There are many types of cells, microorganisms, mediators, and molecules constituting the gut barrier. The physical barrier then contains three major elements which are the intestinal mucosa, intestinal epithelial layer, and microbiota. The central element is the intestinal epithelial layer, which provides physical separation between the lumen and the body. The secretion of various molecules into the lumen reinforces the barrier function on the extraepithelial side, while a variety of immune cells provide additional protection below the epithelial layer. Among all the immune cells, a group of lymphocytes which are termed innate lymphoid cells (ILCs) have been studied heavily in recent years and have important roles and close communications with other cells in the epithelial barrier. In this review, we are going to focus on the interaction and crosstalk among ILCs and other cells in the gut barrier and describe how they influence the barrier function and immune homeostasis.

1.1. First Line of Defense: Gut Barrier Function in Intestinal Physiology. The intestine represents a major gateway for potential pathogens, which also contains antigens from diets and extensive and diverse commensals that need to be tolerated. The gut barrier therefore plays important roles in intestinal physiology such as physical barrier, immune tolerance, pathogen clearance, and chronic inflammation. Its functions rely heavily on a complex group of cells and mediators in the tissue context containing structural cells such as epithelial cells, goblet cells, Paneth cells, and immune cells such as mast cells, dendritic cells, macrophages, and lymphocytes (Figure 1). We will give a brief 


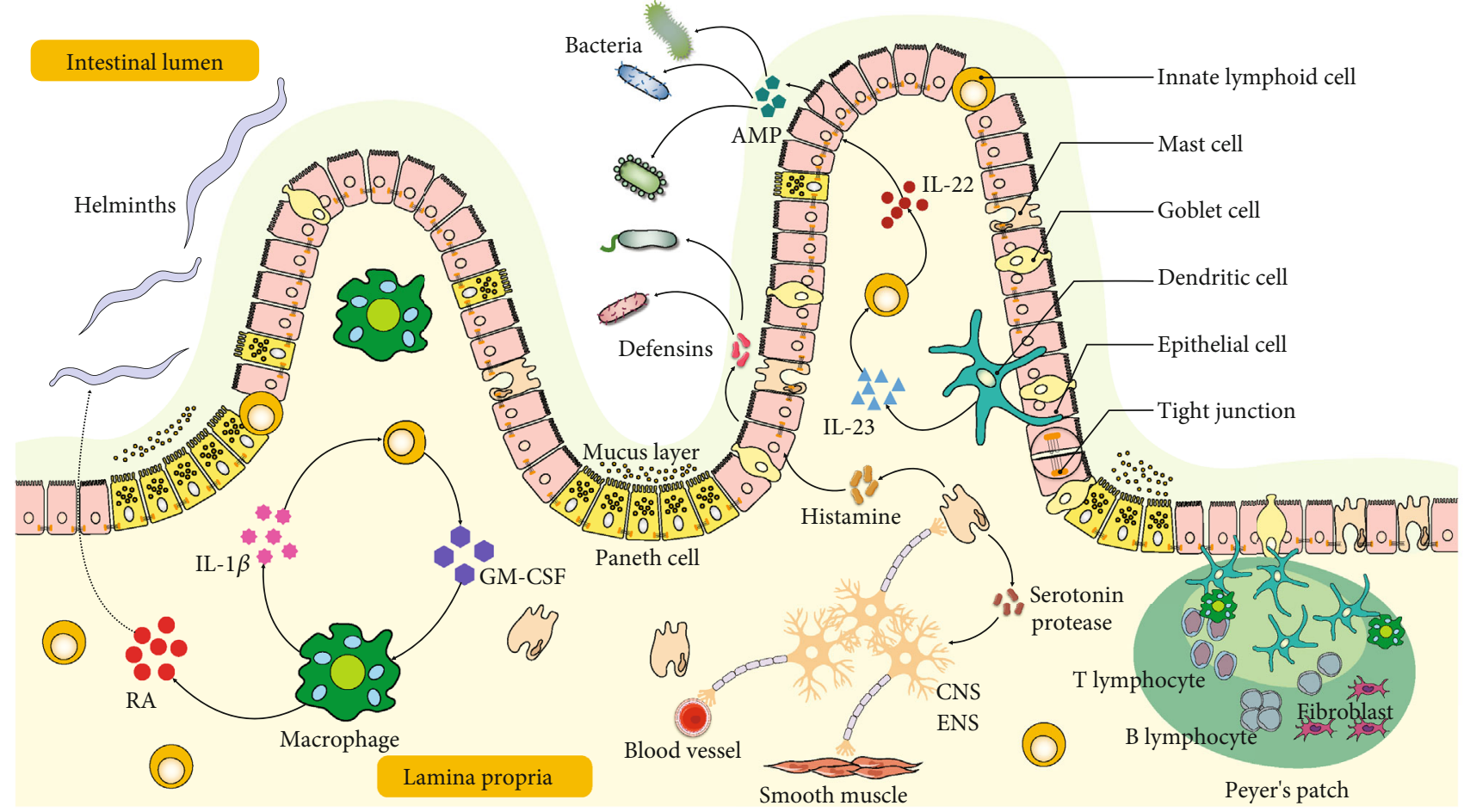

Figure 1: Illustration of intestinal barrier structure and functions. The intestine barrier contains the chemical barrier and the physical barrier. The chemical barrier is composed of antimicrobial peptides (AMPs) such as amphiregulin. It provides chemical agents attacking invading microorganisms including bacteria and helminths. The physical barrier includes the mucus layer and cell junctions between the epithelium. It serves as the wall spatially separating the invading microorganisms and host. There are many types of cells in the gut epithelium regulating the epithelium function. Disruption of the intestinal barrier allows the leak of gut bacteria from the lumen into the lamina propria, inducing excessive immune responses of the host immune cells. Retinoic acid (RA) released by macrophages or dendritic cells assists in host resist helminthic infection. IL-22 released by ILCs promotes epithelial cells secreting AMP in response to bacterial infection, which is regulated by IL-23 from dendritic cells. Moreover, macrophage-derived IL- $1 \beta$ promotes ILCs' production of GM-CSF, which further stimulates more macrophage differentiation from monocytes. The enteric nervous system including neuron and glial cells interacts closely with mast cells and regulates blood vessels. IL: interleukin; AMP: antimicrobial peptide; GM-CSF: granulocytemacrophage colony stimulating factor; RA: retinoic acid; ENS: enteric nervous system; CNS: central nervous system.

description on the role of individual component cells in the gut barrier.

1.2. Intestinal Epithelial Cells. Intestinal epithelial cells constitute the majority of the cellular layer of the gut barrier. The weakening of intercellular junctions between intestinal epithelial cells will result in increased intestinal permeability and systemic exposure to bacterial antigens. The increased diffusion of bacterial components into the blood, lymph, and other extraintestinal tissues is closely related with critical illness, inflammatory bowel disease, celiac disease, food allergy, irritable bowel syndrome, and metabolic syndromes such as diabetes and obesity [2-4]. Therefore, intestinal epithelial permeability provides a novel target for disease prevention and therapy $[5,6]$.

In intact intestines, the intercellular junctions are primary determinants of normal barrier function. There are many kinds of intercellular junctions including the tight junction, adherens junction, gap junction, desmosome, and hemidesmosome. Tight junctions (TJs) are connected areas of the plasma membrane that stitch cells together therefore consisting a series of anastomosing strands. TJs play leading roles in paracellular permeability. Claudins, occludin, and
ZO family proteins are critical components of TJs. Claudins are the most important tetratransmembrane TJs. Their extracellular domains form pores on adjacent cells and regulate $\mathrm{TJ}$ ion selectivity $[6,7]$. Expression levels of the claudin protein are related with the intestinal barrier integrity in different ways depending on the type of claudin isoform [8]. For example, the downregulation of claudins 5 and 8 can drastically reduce the barrier integrity [9]; in contrast, claudin-2, required for the formation of paracellular water channels, is upregulated in IBDs and is highly expressed in leaky epithelial tissues and promotes inflammation [10]. Occludin is the first identified and an important protein for TJ stability. It has a dual role in the intestinal barrier. The expression of occluding was closely correlated with the barrier function both in vitro and in vivo $[8,11]$. Interestingly, genetic manipulated mice which were deprived of occluding showed stability in several epithelial tissues including gastric and intestinal epithelia $[12,13]$. Collectively, the functions of occludin and the mechanism by which occludin regulates the TJ are complex and still remain elusive. Unlike claudins and occludins which are integral membrane proteins and function as a gate, ZOs are peripheral membrane-associated proteins linking membrane protein to the cytoskeleton and ubiquitously 
expressed in epithelial and endothelial cells [14]. The various isoforms, ZO-1, ZO-2, and ZO-3, are all characterized by their ability to interact with different cellular proteins such as claudins or occludins through a multitude of proteinbinding domains, such as the SH3 domain, the PDZ domain, and the leucine-zipper domain. In DSS-induced colitis mouse models, complete loss of ZO-1 expression occurred during the preinflammatory stage [15]. Adherens junctions join the actin filaments of neighboring cells together. Gap junctions are clusters of channels that form tunnels of aqueous connectivity between cells. Desmosomes are even stronger connections that join the intermediate filaments of neighboring cells. Hemidesmosomes connect intermediate filaments of a cell to the basal lamina, a combination of extracellular molecules on other cell surfaces.

1.3. Goblet Cells. Goblet cells secrete mucins which constitute the hydrated gel coated on the luminal surface of the intestinal mucosa. The mucus layer is the front line of innate host defense and prevents large particles and bacteria from coming into direct contact with the underlying epithelium. In the small intestine, the goblet cell-secreted Muc2 mucin, which is the first human secretory mucin to be identified and characterized, constitutes the main component of the mucus layer [16]. The mucin structure is markedly altered in colitis mouse models, and transgenic mice lacking Muc2 gene developed colitis spontaneously [17]. Besides the secretory mucin glycoproteins (MUC2), goblet cells synthesize many bioactive molecules such as epithelial membranebound mucins (MUC1, MUC3, and MUC17), trefoil factor peptides (TFF), resistin-like molecule $\beta(\operatorname{RELM} \beta)$, and Fc- $\gamma$ binding protein (Fcgbp) [18].

Mucin secretion is frequently coupled with increased synthesis of mucins. The biology of mucin compositions and syntheses have been summarized in details [19]. Activation of mucin synthesis can be induced Th1 cytokines (e.g., tumor necrosis factor- (TNF-) $\alpha$ ) and Th2 cytokines (e.g., interleukin- (IL-) 4, IL-13), microbial products (e.g., lipopolysaccharide (LPS)), and neuropeptides. The regulation of mucin expression is controlled either by transcriptional regulation or by epigenetic regulation [18]. Due to the potent binding site in MUC2 promoters, cumulative evidences indicate that transcriptional regulation of MUC2 is mediated by transcription factor nuclear factor- (NF-) $\kappa \mathrm{B}$, a common activated transcription factor during inflammation in the gastrointestinal tract $[20,21]$, intestine-specific transcription factors Cdx-1 and Cdx-2 [22], forkhead box transcription factors Foxa1 and Foxa2 [23], and CREB/ATF1 [24]. Epigenetic regulation includes DNA methylation, histone modifications, and microRNA silencing. MUC2 gene expression is regulated closely by DNA methylation and histone modifications in the $5^{\prime}$ flanking region of MUC2 promoter [25]. In mucinous and nonmucinous colorectal cancer tissues, MUC2 expression is downregulated by methylation of $\mathrm{CpG}$ islands in the specific regions of MUC2 promoter [26].

1.4. Paneth Cells. Paneth cells reside mainly in small intestine epithelium and are located at the base of crypts of
Lieberkühn (just below the intestinal stem cells in the intestinal glands) and contribute to intestinal innate immunity by secreting a diverse repertoire of antimicrobial peptides and proteins [27].

Paneth cells are vital in controlling intestinal barrier penetration by commensal and pathogenic bacteria. They sense enteric bacteria through cell-autonomous MyD88dependent toll-like receptor (TLR) activation, triggering expression of multiple antimicrobial factors such as lysozyme and defensins (called cryptdins in mice) [28]. Defensins are the principal molecules secreted by Paneth cells. Defensins have a hydrophobic domain which can interact with phospholipids on bacterial cell membranes and thus lead to bacteria cell lysis. Paneth cells are daughter cells differentiated from intestinal stem cells [27]. Interestingly, a recent ex vivo study by Dr. Han Clevers group showed that Paneth cells lose their secretory expression signature, reenter the cell cycle, and acquire stem-like properties, contributing to the tissue regenerative response to inflammation [29].

1.5. Mast Cells. Mast cells in the gastrointestinal (GI) tract are located in close proximity to sensory nerve fibers, which by communicating bidirectionally play roles in the brain-gut axis [30, 31]. The interactions between mast cells and enteric neurons ensure the function of the enteric nervous system (ENS) regulation of the GI tract physiology such as motility, secretion, and microcirculation as well as immune responses $[32,33]$. Moreover, the interactions are closely correlated with severity and frequency of GI tract disorders such as abdominal pain [34]. Mast cells in the GI tract comprise 1$5 \%$ of mononuclear cells in the lamina propria, submucosa, and epithelial layers [30]. Mast cells are derived from the myeloid stem cells and are similar to granulocytes. They exert their functions in two steps, which contain activation inducing degranulation and release of inflammatory mediators, including histamine, cytokines, proteoglycans, and proteases [35]. They contribute to innate and acquired immunities and are important effector cells in host defense in GI tracts overloaded every day with external stimuli such as food, pathogens, toxic substances, commensal flora, and moreover endogenous small molecules such as neurotransmitters, neuropeptides, growth factors, and hormones. Generally, mast cell activation is classically stimulated by interaction of antigens coming from allergens with its specific IgE antibody bound to the mast cell membrane through the highaffinity receptor FceRI [36]. Besides, mast cells also express receptors for IgG $(\mathrm{FcRI})$, immunoglobulin free-light chains (IgLCs), other Ig-associated receptors, complement fractions, and toll-like receptors. Activation via one of these receptors results in phosphorylation cascades and activation motifs that lead to intracellular calcium flux, activation of transcription factors such as activator protein 1 (AP-1), microphthalmia-associated transcription factor (MITF), and signal transducers and activators of transcription 5 (STAT-5) and downstream protease, cytokines, and mediator expression $[30,32]$. Based on different protease contents, most mast cells can be divided into two categories: $\mathrm{MC}_{\mathrm{T}}$ containing mainly tryptase and $\mathrm{MC}_{\mathrm{TC}}$ containing 
tryptase, chymase, and carboxypeptidases [33, 37]. In the GI tract, $\mathrm{MC}_{\mathrm{T}}$ comprise $\sim 98 \%$ of all mast cells in the mucosa and $\sim 13 \%$ of all mast cells in the submucosa [33].

1.6. Dendritic Cells. Dendritic cells (DCs) are key modulators that shape the immune system. In mucosal tissues, DCs play surveillance roles to sense infection and also function as the major antigen-presenting cells that stimulate the differentiation of naive $\mathrm{T}$ cells. They function in bridging the innate signaling and adaptive immune systems to maintain the homeostasis of the intestinal immune environment [38]. Besides, DCs are able to open tight junctions and to sample antigens directly across the epithelium both in vivo and in vitro [39]. Intestinal DC can be divided into several subsets based on the surface expression of integrins CD11c and CD103. More recently, CD24 and Sirp $\alpha$ have been introduced for better discrimination of DCs from macrophages [40].

Although DCs are located primarily in lamina propria and mucosa-associated lymphoid tissues rather than in the epithelial barrier, DCs have intimate interactions with the epithelial layer. Goblet cells were shown to transfer small soluble antigens from the intestinal lumen to CD103+ DC [41]. Chemokines secreted by enterocytes in response to TLR ligand exposure can induce the above-mentioned relocation of lamina propria DC to the epithelium [42]. Epithelial and stromal cells secrete factors, which are thought to induce DC tolerance, such as RA, TGF- $\beta$, PGE-2, and TSLP [43]. Establishing intestinal tolerance is critical for the prevention of intestinal diseases such as IBD, and manipulating mucosal DCs provides potential therapeutic strategies to protect against infectious diseases.

1.7. Macrophages. The intestine contains the largest pool of macrophages in the body. Located in the subepithelial lamina propria, intestinal macrophages are the most abundant mononuclear phagocytes. They maintain mucosal homeostasis by capturing and eliminating bacteria that cross the epithelial barrier and meet the constant phagocytosis need for epithelial renewal $[43,44]$. They are important components of protective immunity and are involved in the pathology of inflammatory bowel disease (IBD) [45]. Macrophagerestricted IL-10 receptor deficiency causes severe spontaneous colitis [46]. Mouse model genetically inactivation of stat3 in macrophages will develop inflammation in the colon spontaneously and tumor lesions including invasive carcinoma with a frequency similar to that observed in human IBD patients [47].

Defining the biological roles of intestinal macrophages, characterizing the phenotype, and defining the origins of different populations of myeloid cells in the mucosa have been studied quite extensively recently [45]. Intestinal macrophages originate from yolk sacs or fetal livers at the embryonic stage and are replaced in the gut by Ly6C ${ }^{+}$blood monocytes shortly after birth [48]. In adult guts, they undergo continuous renewal from monocytederived cells. In the process differentiation, monocytes lose Ly6C expression while other macrophage surface markers are upregulated such as MHCII, F4/80, CD11c, and CX3CR1 [43, 49].

While it has been known for many years that macrophages are present in deeper layers of the gut wall, only recently has work begun to interrogate their role in intestinal homeostasis [44]. Macrophages are also found in the submucosa, and recent depletion studies have revealed a role for these cells in maintaining the integrity of the submucosal vasculature [50].

1.8. Intraepithelial Lymphocytes. The intraepithelial lymphocytes (IELs) that reside between the intestinal epithelial cells (IECs) form one of the main branches of the immune system [51]. The small intestine contains approximately 1 IEL per 10 intestinal epithelial cells (IECs), and this ratio is lower in the colon [52]. IELs are resident in the intestinal epithelium and do not recirculate [53]. They express several characteristic surface receptors such as the chemokine receptor CCR9, which interacts with CCL25 produced by IECs and thus assists in recruiting IELs to the gut mucosa [52]. Intestinal IELs also express integrin $\alpha \mathrm{E} \beta 7 \quad\left(\alpha_{\mathrm{E}}\right.$ is also known as CD103), which interacts with E-cadherin on enterocytes to facilitate entry and retention in the intestinal epithelium. Approximately $90 \%$ of all IELs express $\mathrm{T}$ cell receptors (TCRs), and these cells have been the main focus of studies on IEL biology.

1.9. Neurons. Intestinal neurons can be classified as intrinsic and extrinsic. The former can also be termed as enteric neurons which have cell bodies within the gut, while the latter refers to neurons which have cell bodies located outside the intestine such as sympathetic and parasympathetic autonomic nervous systems [54]. The intestine is the largest immune cell compartment with millions of enteric neurons in the body. Therefore, it is also called the second brain [55]. Enteric neurons include myenteric and submucosal neurons. Submucosal neurons control gut secretions, nutrient absorption, and local blood flow whereas myenteric neurons orchestrate smooth muscle contractions $[56,57]$ (Figure 1).

Apart from enteric neurons, there are enteric glial cells found in enteric ganglia in lamina propria and smooth muscle [54]. They outnumber enteric neurons. Together, they constitute the enteric nervous system (ENS), which could continuously extend from the base of the crypts to the mucosa. Glial cells are vital to intestinal barrier integrity. Complete deletion of glial cells leads to fatal jejunoileitis in mice due to barrier integrity disruption [58, 59]. However, partial conditional depletion of enteric glial cells failed to induce inflammation and barrier disruption in intestines [60]. Enteric glial cells participate in sensing pathogens and produce neurotrophic factors and help maintain the epithelial barrier integrity. Finally, when neurons are damaged, enteric glial cells can transdifferentiate into enteric neurons to compensate [54].

1.10. Innate Lymphoid Cells in the Gut Barrier. Innate lymphoid cells (ILCs) are a relatively recently discovered 
TABLE 1: List of some important studies in ILC research history.

\begin{tabular}{|c|c|c|}
\hline Years & Events & Reference \# \\
\hline 1975 & Discovery of NK cells as the first subsets of ILCs & [63] \\
\hline 1997 & $\begin{array}{l}\text { Discovery of LTi cells (later defined as one subset of ILC3s) which are } \\
\text { essential for the development of lymph nodes during embryogenesis }\end{array}$ & [64] \\
\hline 2006 & Characterization of GATA3 and CD127 on ILC2s & [65] \\
\hline 2009 & $\begin{array}{l}\text { ILC3s are the source of endogenous IL-22 and constrain } \\
\text { inflammation at the mucosal site }\end{array}$ & [66] \\
\hline 2010 & $\begin{array}{l}\text { Identification of ILC2s in mice that produce type } 2 \text { cytokines and } \\
\text { contribute to antihelminth immunity and type } 2 \text { inflammation }\end{array}$ & [67] \\
\hline 2010 & Identification of a role for ILC3-like cells in promoting intestinal inflammation & [68] \\
\hline 2011 & $\begin{array}{l}\text { First description of a tissue-protective role for ILC2s, describing how ILC2s } \\
\text { produce amphiregulin, a ligand of EGFR, and contribute to lung-tissue } \\
\text { repair following influenza A virus infection in mice }\end{array}$ & [69] \\
\hline 2013 & $\begin{array}{l}\text { First evidence of non-NK cell ILC1s in humans and the transcription } \\
\text { factor T-bet responsible for ILC1s differentiation }\end{array}$ & {$[70,71]$} \\
\hline 2013 & First evidence that ILC3s directly regulate adaptive immune responses & [72] \\
\hline 2013 & Experts described consensus nomenclature for ILC subsets & [73] \\
\hline
\end{tabular}

lymphocytes compared to its other counterpart lymphocytes such as Th cells or Th17 cells. ILCs do not express the type of diversified antigen receptors expressed on T cells and B cells, and they are largely tissue-resident cells and are deeply integrated into the residential tissues [61]. While adaptive lymphocytes are most numerous in lymphoid organs-hence the derivation of the term "lymphocyte"-ILCs are relatively rare in primary and secondary lymphoid tissues. Consequently, their existence has been overlooked for many years, as immunologists focused efforts on peripheral blood and lymphoid organs. However, it is now recognized that their positioning in peripheral tissues particularly abundant at barrier surfaces in the lung, skin, and intestinal tract affords a strategic advantage for ILCs as early responders to tissue perturbation. Indeed, as a result of their location and effector phenotype, ILCs are rapid-responding cells and they produce cytokines within hours of activation, in contrast to the days required for naive adaptive lymphocytes to be primed, expand, differentiate, and enter tissues [62]. Table 1 lists some of the remarkable findings in ILC research history about ILC discovery, identification, and functions.

ILCs have been identified with many subtypes mainly divided into three groups. Group 1 ILCs include noncytotoxic ILC1s and cytotoxic conventional NK cells. Conventional NK cells were first discovered in 1975 and have been studied well with a longer history compared to other types of ILCs. Many of their functions, interaction with microbiota, antitumor responses, and involvement in the gut barrier have been investigated and reviewed in details elsewhere [74, 75]. Hence, we are not going to review this part here. We will term group 1 ILCs as ILC1s thereafter. ILC1s are regulated by Tbet and can produce IFN- $\gamma$, GM-CSF, granzyme, and perforin in response to IL-12, IL-18, or other activators such as pathogens or tumors. They cooperate with Th1 cells against intracellular microbes such as viruses, bacteria, or parasites [71, 73, 76, 77]. Group 2 ILCs (ILC2s), similarly to Th2, express Gata3 and can produce IL-4, IL-5, IL-13, IL-9, and amphiregulin in response to IL-25, IL-33, and TSLP (thymic stromal lymphopoietin). ILC2s are essential in the immune response against large extracellular parasites and allergens. Their production of antimicrobial peptides promotes tissue damage repair $[78,79]$. A recent discovery published by Huang et al. on Science by using mouse models and advanced imaging techniques to monitor ILC activation and movement showed that ILC2s originate in the gut, enter lymphatic vessels, circulate in the bloodstream, and can migrate to other organs to help fight infection against helminth [80]. The trafficking of ILC2s is in a partly sphingosine 1-phosphate(S1P-) dependent manner [80]. Group 3 ILCs (ILC3s), mirroring Th17, express ROR $\gamma$ t, the lymphotoxins $\alpha$ and $\beta$, IL-17 and IL-22, GM-CSF, and TNF- $\alpha$. They can be activated by IL- 23 and IL- $1 \beta$ or by NCR ligands, and they combat extracellular microbes, such as bacteria and fungi [77] (Table 2).

The classification of ILCs into ILC1, ILC2, and ILC3 subsets is a simplified theoretical approach for understanding ILC diversity. ILC function and differentiation programs are more complicated during immune responses. Heterogeneity and plasticity of ILCs have been identified in both human and mouse studies. Tissue-resident T-bet+ ILC1s may derive from four sources in humans: ILC precursors (ILCP); converted ILC2s exposed to IL-12 and IL-1 $\beta$; converted ILC3s exposed to IL-2, IL-15, and IL-23; and NK cells exposed to TGF- $\beta$ [81, 82]. Bernink and colleagues reported bidirectional plasticity between ILC1 and ILC3 in the intestinal lamina propria with different environment stimuli such as IL-23, IL-1 $\beta$, retinoic acid, or dendritic cells [83].

\section{Crosstalk between Innate Lymphoid Cells and Other Immune Cells}

\author{
2.1. Crosstalk between Innate Lymphoid Cells and \\ Dendritic Cell
}


TABLE 2: Innate lymphoid cells in the gut.

\begin{tabular}{|c|c|c|c|c|c|c|}
\hline \multirow{2}{*}{ Types } & \multicolumn{2}{|c|}{ Surface marker } & \multirow{2}{*}{ Stimulus } & \multirow{2}{*}{$\begin{array}{c}\text { Regulatory } \\
\text { transcription factor }\end{array}$} & \multirow{2}{*}{ Signature released cytokines } & \multirow{2}{*}{$\begin{array}{l}\text { Functions in the } \\
\text { gut barrier }\end{array}$} \\
\hline & Mouse & Human & & & & \\
\hline \multirow{5}{*}{ ILC1 } & CD160 & CD103 & \multirow{5}{*}{ IL-12, IL-15 } & \multirow{5}{*}{ T-bet } & \multirow{5}{*}{$\begin{array}{l}\text { IFN- } \gamma, \text { TNF- } \alpha, \text { GM-CSF, } \\
\text { granzyme, perforin }\end{array}$} & \multirow{5}{*}{$\begin{array}{c}\text { Defense against virus, } \\
\text { pathogens }\end{array}$} \\
\hline & NKp46 & CD160 & & & & \\
\hline & NK1.1 & CD56 & & & & \\
\hline & & NKp46 & & & & \\
\hline & & NKp44 & & & & \\
\hline \multirow{5}{*}{ ILC2 } & IL17RB & IL17RB & \multirow{5}{*}{ IL-25, IL-33, TSLP } & \multirow{5}{*}{ GATA3 } & \multirow{5}{*}{$\begin{array}{l}\text { IL-4, IL-5, IL-13, IL-9, } \\
\text { amphiregulin }\end{array}$} & \multirow{5}{*}{ Helminth expulsion } \\
\hline & IL-33R & IL-33R & & & & \\
\hline & $\mathrm{CD} 25$ & $\mathrm{CD} 25$ & & & & \\
\hline & CD127 & CD127 & & & & \\
\hline & & CRTH2 & & & & \\
\hline ILC3 & NKp46 & NKp44 & $\begin{array}{l}\text { IL-1 } \beta \text {, IL-23, } \\
\text { NCR ligand }\end{array}$ & $\mathrm{ROR} \gamma \mathrm{T}$ & IL-17, IL-22, GM-CSF, TNF- $\alpha$ & $\begin{array}{l}\text { Defense against } \\
\text { bacteria, fungi }\end{array}$ \\
\hline
\end{tabular}

2.1.1. ILC1s and DCs. ILCs are characterized by prompt response after infection or injury. Tissue-resident ILC1 confer early host protection at initial sites of viral infection [84]. In a mouse model infected with pathogenic DNA viruses, Wong et al. have found that migratory dendritic cells (mDCs) induce expression of NKG2D ligands after sensing the double-stranded DNA virus via TLR9/MyD88 and promote IFN- $\gamma$ expression in classical NK cells and group 1 ILC (mainly NK cells) already in draining lymph nodes (dLNs) through NKG2D (Figure 2). Inflammatory monocytes are also recruited to dLNs by mDCs in a TLR9/MyD88-dependent manner responding to IFN- $\gamma$ [85].

2.1.2. ILC2s and DCs. Crosstalk between ILC2s and DCs is believed to be necessary in the host to combat parasitic helminth infection executed by type 2 immune responses [78]. DCs are well-defined for antigen presentation and type 2 chemokine production during the memory Th2 cell recallresponse, and it is also known that DCs can be stimulated by type 2 cytokines to produce chemokines CCL17 and CCL22, which attract its cognate-receptor CCR4-expressing memory TH2 cells. ILC2s act upstream of DCs and are essential for their production of memory $\mathrm{TH} 2$ cell chemoattractant CCL17. At the barrier sites, ILC2s respond to helminth infection and become activated by alarmins including IL-25, IL-33, and TSLP secreted by epithelium in the gut as an important early cellular event and produce high amounts of type 2 cytokines [86] (Figure 2). Halim et al. have reported that ILC2s-produced IL-13 has been linked to the migration of DCs in allergic asthma [87]. This interaction has been extended by Oliphant et al. that ILC2s and T cells cooperate through MHCII-dependent activation to promote DC migration to the draining lymph nodes to potentiate the Th2 generation from naïve T cells against helminth infection [78]. However, how IL-13 controls the migratory function of DCs still remains elusive.

2.1.3. ILC3 and DCs. The interactions between ILC3 and DCs are discussed below in ILC3 and Macrophages.

\subsection{Crosstalk between Innate Lymphoid Cells and Macrophage}

2.2.1. ILC1 and Macrophages. Studies on ILC1 and macrophages in intestinal tract have been scarce. Recent studies in inflammatory bowel disease animal models and intestinal infection with parasites such as Toxoplasma gondii have shown that ILC1s secrete IFN- $\gamma$ and TNF- $\alpha$ and contribute to the inflammatory response and pathology in response to IL-12 and IL-15 together with macrophages [71, 88-90] (Figure 3(a)). However, studies of their interactions in obesity have shown promise. ILC1 displayed cytotoxic activity toward adipose tissue macrophages. During obesity, this killing ability was impaired and ILC1s were reported to be the major contributors for IFN- $\gamma$ upregulation resulting in the expansion of proinflammatory M1 macrophages, and this could lead to the accumulation of pathogenic proinflammatory macrophages [91]. This interaction contributes to M1 macrophage polarization and systemic insulin resistance [92].

2.2.2. ILC2s and Macrophages. ILCs can promote plastic macrophages to differentiate into alternatively activated macrophages (or M2 macrophages) in some helminth infection models to provide protective functions and tissue repair responses against helminth infection [93]. IL-25- or IL-33-activated ILC2s were found to promote M2 polarization and Treg cell expansion contributing protective immunity [94]. IL-33-activated ILC2s induced M2 polarization through IL-4 receptor signaling and directly regulated beige fat biogenesis [95]. IL2Cs has also been described to promote M2 macrophage accumulation in visceral adipose tissue during helminth infection [96, 97] (Figure 3(b)). In an airway barrier, alveolar macrophages can secrete IL-33 which will elicit direct activation of ILC2cs to produce substantial amounts of IL-13 [97]. This crosstalk in gut barriers needs to be confirmed.

2.2.3. ILC3 and Macrophages. Intestinal mucosal tissueresident macrophages together with DCs are the two main 


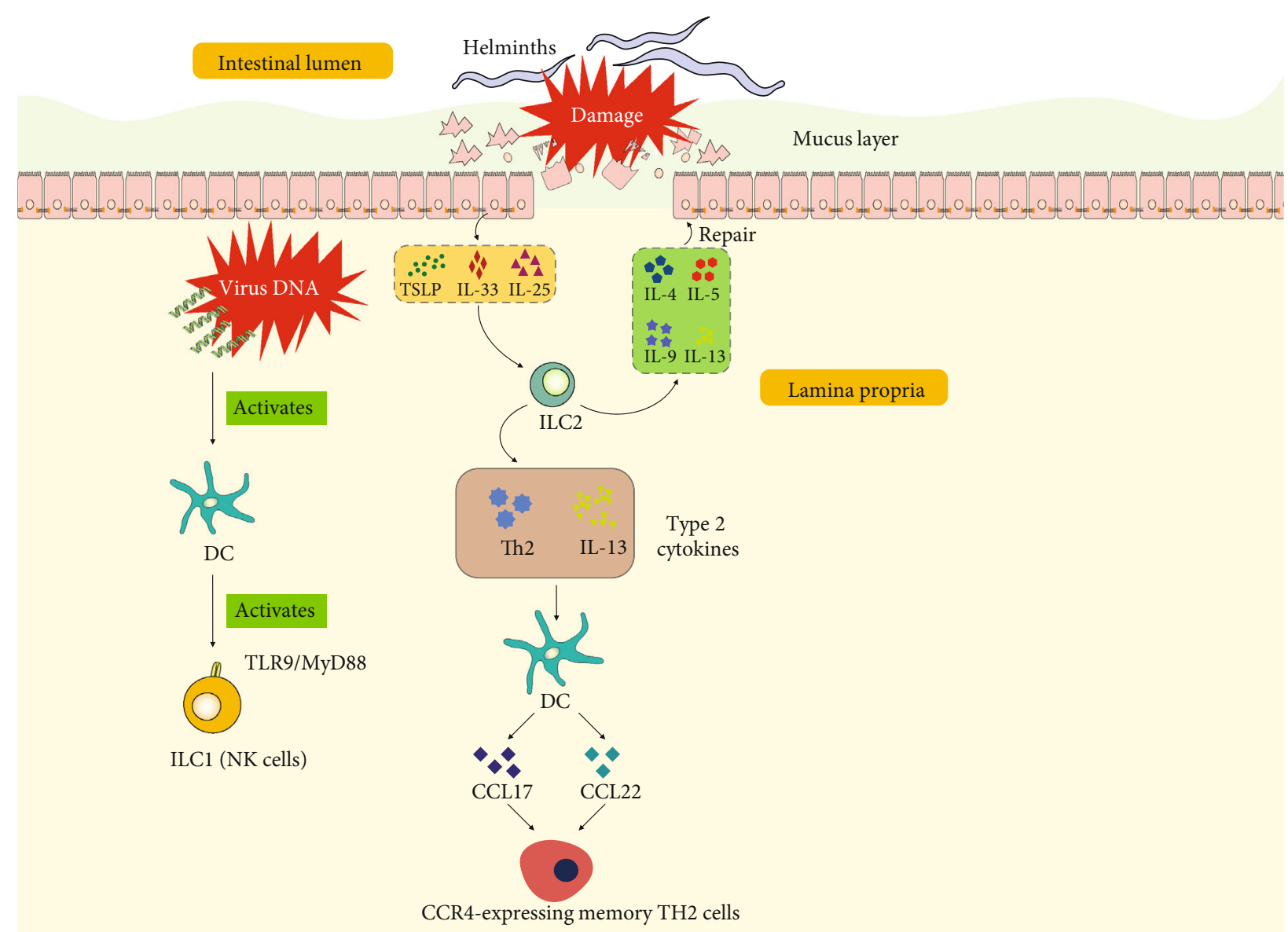

FIGURE 2: Illustration of interactions of ILCs and DCs in the intestinal tract. At the initial site of viral infection, virus DNA activates DCs, further activating ILC1 (mainly NK cells) by the TLR9/MyD88 pathway. After damage caused by helminths, epithelial cells produce TSLP, IL-33, and IL-25. These cytokines stimulate ILC2s producing type 2 cytokines including IL-4, IL-5, IL-9, and IL-13 which participate in repair of epithelial cells and mucus layer. In addition, ILC2-derived Th2 and IL-13 stimulate DCs, inducing the release of CCL17 and CCL22 and recruitment of CCR4-expressing memory TH2 cells. IL: interleukin; TSLP: thymic stromal lymphopoietin; DC: dendritic cell; ILC: innate lymphoid cell; CCL: chemokine (C-C motif) ligand; Th: helper T cell.

cell populations to detect microbial signals and to capture and process extracellular antigens. Meanwhile, macrophages and DCs contribute to the maintenance of immune tolerance by the induction or expansion of FoxP3 ${ }^{+}$Treg cells in the intestine by producing retinoic acid (RA). GM-CSF (or Csf-2) is needed to maintain DCs and macrophage numbers in the colon as well as for the Treg cells. A seminal work by Mortha et al. demonstrated that ROR $\gamma \mathrm{t}^{+}$innate lymphoid cells (ILC3s) are the primary source of GM-CSF in the gut and that ILC-driven GMCSF production was dependent on the ability of macrophages to sense microbial signals [98]. Macrophages detect microbial signals through a TLR-MyD88-dependent manner and produce interleukin- $1 \beta$, which can act on ILC3s [98] (Figure 3(c)).

\subsection{Crosstalk between Innate Lymphoid Cells and Epithelial Cells}

2.3.1. ILC1s and Epithelial Cells. ILC1s are enriched in the upper GI tract [99]. In murine models, ILC1s protect epithe- lial cells. Helicobacter typhlonius is commensal in the murine microbiota that closely resembles Helicobacter pylori, the frequent colonizer of the human stomach associated with gastritis, peptic ulcer, and gastric cancer. Mice lacking Tbet, the transcription factor controlling ILC1s differentiation, develop colitis triggered by Helicobacter typhlonius [100]. This result shows that ILC1 participate in the defense against bacterial infection. During pathological bacteria Salmonella infection at the intestinal tract, ILC1s are the main source of IFN- $\gamma$, which drives the secretion of mucus-forming glycoproteins required to protect the epithelial barrier [101].

2.3.2. ILC2s and Epithelial Cells. ILC2 activation is dependent on epithelial-derived cytokines, such as IL-25, IL-33, and TSLP [97], prostaglandin D2 (PGD2) [102], and leukotriene D4 [103]. After activation, ILC2 secrete type 2 cytokines such as IL-4, IL-5, IL-9, and IL-13, which have tissue repair functions and will eventually protect epithelial cells [97].

2.3.3. ILC3 and Epithelial Cells. ILC3s protect the intestinal epithelial cells and maintain the homeostasis against various 


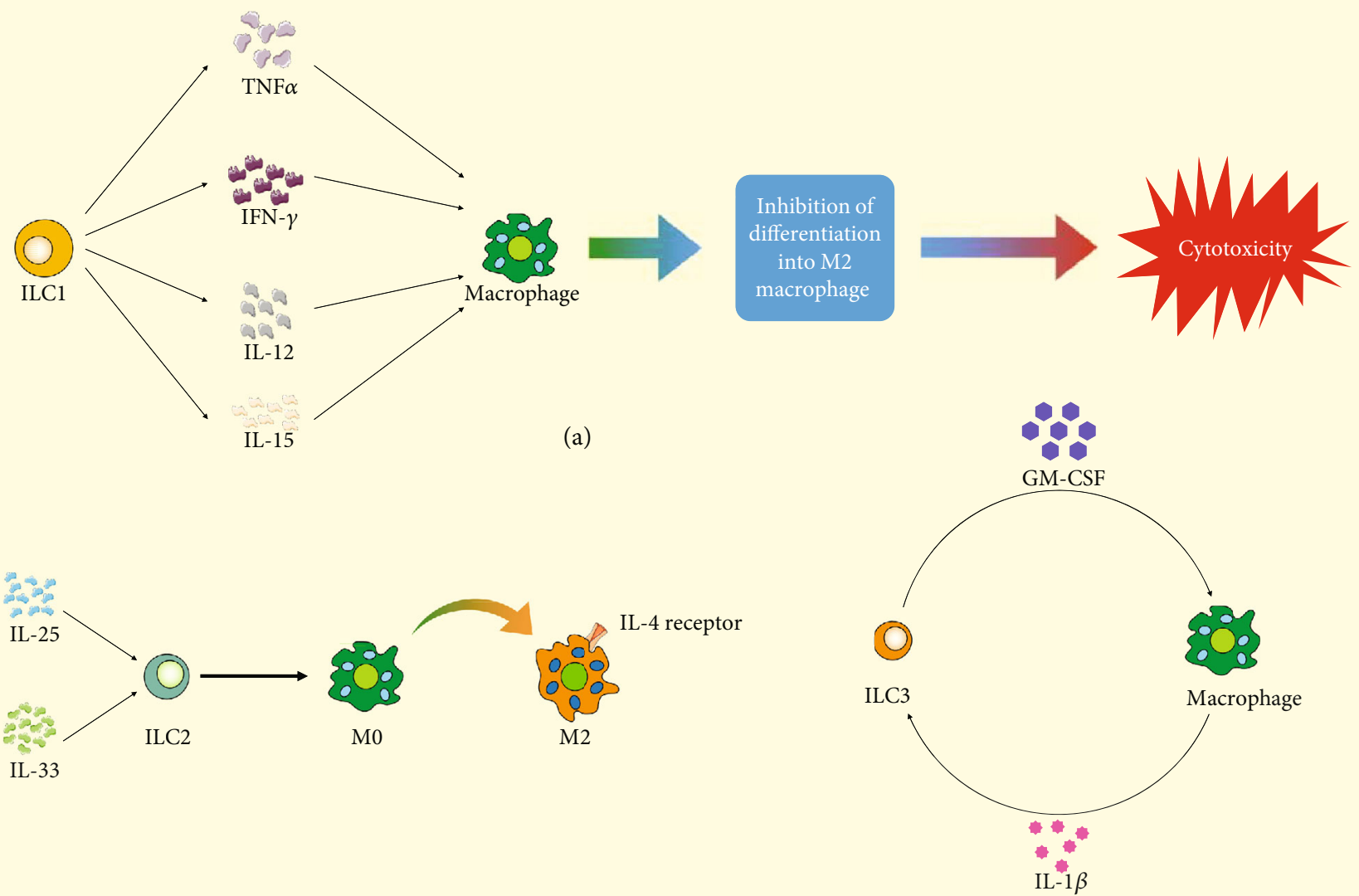

(b)

(c)

FIGURE 3: Illustration of interactions of macrophages and different ILCs. (a) TNF- $\alpha$, IFN- $\gamma$, IL-12, and IL-15 released from ILC1 stimulate macrophage differentiating into cytotoxic macrophage and inhibit differentiation into M2 macrophage differentiation. (b) After being stimulated by IL-25 and IL-33, ILC2 promotes the transformation of M2 macrophage from M0 macrophage. (c) The crosstalk of ILC3 and macrophage is mainly induced by GM-CSF and IL- $1 \beta$. ILC: innate lymphoid cell; TNF: tumor necrosis factor; IL: interleukin; M0: M0 macrophage; M2: M2 macrophage; GM-CSF: granulocyte-macrophage colony stimulating factor.

pathogens. The protective role of ILC3s on epithelial cells is fulfilled by signature cytokine IL-22 released by ILC3s. Upon activation, ILC3s secrete IL-22, IL-17, and GM-CSF. IL-22 is a member of the IL-10 family and displays a homologous secondary structure, binding to its heterodimeric receptors IL-22R1 and IL-10R2 on epithelial cells. IL-22 signaling orchestrates the production of mucin and mediates epithelial cell proliferation and survival upon infection [104]. Mechanistically, IL-22 promote the production of nucleotide oligomerization domain-containing protein 2 (NOD2), which functions as a mammalian cytosolic pathogen recognition molecule. NOD2 associates with the caspase activation and recruitment domain of RIP-like interacting caspase-like apoptosis regulatory protein kinase (RICK)/RIP2 and activates nuclear factor- (NF-) $\kappa$ B in epithelial cells [105]. The activation of NF- $\kappa \mathrm{B}$ induces epithelial cells to produce antimicrobial peptides and mucin. Moreover, ILC3-derived IL-22 can induce STAT3 phosphorylation and activate $\operatorname{Lgr} 5^{+}$intestinal stem cells for epithelial regeneration to impede tissue damage $[106,107]$ (Figure $4(\mathrm{a})$ ). In addition, ILC3s protect epithelial cells from gut bacteria by adjusting intestinal epithelial cell glycan metabolism. ILC3s have been reported to induce the expression of fucosyl- transferase 2 (Fut2), which catalyze fucosylation in intestinal epithelial cells in mice [108]. This induction requires the cytokines IL-22 and lymphotoxin produced by ILC3s. Fucosylation is a major mechanism of commensal bacteria utilizing dietary carbohydrate in the host. Disruption of intestinal fucosylation results in increased susceptibility to infection by pathological bacteria such as Salmonella typhimurium [108] (Figure 4(b)).

2.4. Crosstalk between Innate Lymphoid Cells and GutAssociated Lymphoid Tissue (GALT). GALT is a major component of the mucosa-associated lymphoid tissue (MALT) in the gut. It is the sensor for luminal content and is critical to lymphoid maturation, activation, and differentiation. It comprises isolated and aggregated lymphoid follicles, cryptopatches (CPs), and tertiary lymphoid tissue. ILCs play a central role within GALT. Prenatal GALT development is dependent on ILC lymphoid-inducer function. Postnatally, these cells rapidly respond to commensal and pathogenic intestinal bacteria, parasites, and food components by polarized cytokine production such as IL-22, IL-17, or IL-13 and further contribute to GALT formation and function [109]. 


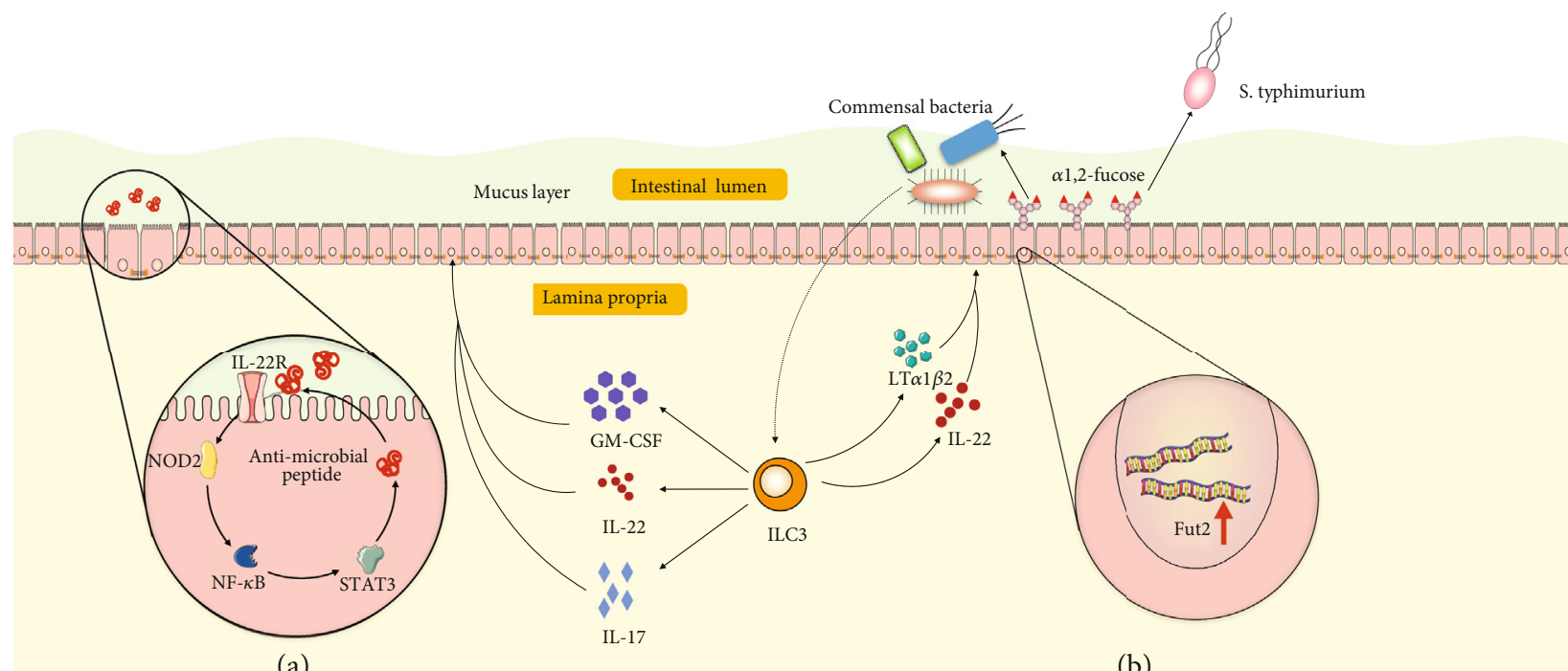

(a)

(b)

FIGURE 4: Illustration of epithelial cells and ILC3 interactions. (a) After bacterial infection, ILC3-derived GM-CSF, IL-22, and IL-17 stimulate epithelial cells by activating the IL-22R-NOD2-NF- $\kappa$ B signaling pathway and result in epithelial cells secreting antimicrobial peptides into the mucus layer. (b) ILC3-derived IL-22 and lymphotoxin (LT $\alpha 1 \beta 2$ ) induce fucosyltransferase 2 (Fut2) gene expression in epithelial cells and result in fucose production in the intestinal tract. Fucose can be utilized by commensal bacteria but not by pathological bacteria such as Salmonella typhimurium. ILC: innate lymphoid cell; GM-CSF: granulocyte-macrophage colony stimulating factor; NOD2: nucleotide oligomerization domain-containing protein 2 ; NF- $\kappa$ B: nuclear factor- (NF-) $\kappa$ B; STAT3: signal transducers and activators of transcription 3.

2.5. Crosstalk between Innate Lymphoid Cells and Neurons in the Gut. At mucosal barriers, ILCs reside in close proximity to neurons and glial cells, and the crosstalk composes the functional neuron-ILC units $[54,110]$.

In response to helminthic infection, intestinal cholinergic neurons regulate ILC2 function via production of neuromedin $\mathrm{U}(\mathrm{NMU})[111,112]$. NMU signals through NMU receptor 1 (NMUR1) expressed in ILC2s and leads to a rapid and potent production of type 2 inflammatory cytokines, IL- 5 and IL-13, and of the tissue-protective cytokine amphiregulin (Figure 5) $[111,112]$. In vivo activation of this signaling axis enhances ILC2 responses and confers immediate tissue protection against helminthic infection. Subsequently, neuron-ILC2 units were identified as part of a neuron-based regulatory circuit that dampens ILC2-mediated type 2 inflammation [113]. ILC2s express the $\beta 2$-adrenergic receptor and colocalize with adrenergic neurons in the intestine. Abrogation of $\beta 2$-adrenergic receptor-mediated signaling resulted in increased ILC2 responses, type 2 inflammation, and lower helminth infection burden, effects that were reversed by $\beta 2$-adrenergic receptor agonist treatment [113]. Together, these studies demonstrate that ILC2s can integrate the cholinergic and sympathetic neuronal pathways to fulfill complex regulatory functions against helminth infection.

A cutting-edge study by Ibiza et al. revealed that enteric ILC3s are part of neuroglia-ILC3 units which are orchestrated by neurotrophic factors [114]. Enteric glial cells sense microbial and host alarmin cues, which leads to increased glia-derived production of neurotrophic factors that in turn induce IL-22 production by RET (a receptor for neurotrophic factors)-expressing ILC3s. Consequently, this glia-ILC3 axis is necessary for intestinal tissue repair upon inflammatory and infection insults [114].

\section{Regulation of Innate Lymphoid Cells and Pharmacological Potentials in Intestinal Innate Immunity}

Due to the close interactions with other cells and prompt response to enteric bacteria or injury in the intestinal tract, intestinal ILCs may be targeted to manipulate immune responses early during vaccination, immunotherapy, and inflammatory pathology. Therefore, it is imperative to study comprehensively the fundamental molecular signals that regulate ILC diversity and functions. Although ILC-specific targets have not yet been identified, the activation pathways and effector molecules that can modulate ILC may provide potential therapeutic benefits.

\subsection{ILC Transcriptional Checkpoint Targeting Strategy.}

Based on the transcription factors that govern the cell differentiation, function, and signature cytokine production, ROR $\gamma$ t inhibitors have been identified primarily to block Th17-mediated inflammatory pathology [115-117]. These inhibitors can be used to block ILC3s as well, although there is a study showing that inhibition of ROR $\gamma$ t selectively targets IL-17 producing iNKT and $\gamma \mathrm{t}-\mathrm{T}$ cells but not IL-22-expressing cells [118]. Similar strategies targeting the important transcriptional checkpoint may be followed such as modulation of the activity of NK cells and ILC1s by targeting T-bet [119]. However, selective loss of T-bet in ILC1s leads to the expansion and increased activity of ILC2s [120]. The controversy or unexpected results demonstrate that we still need to study comprehensively the functions and signaling pathways that regulate the pathogenic or protective immune responses. 


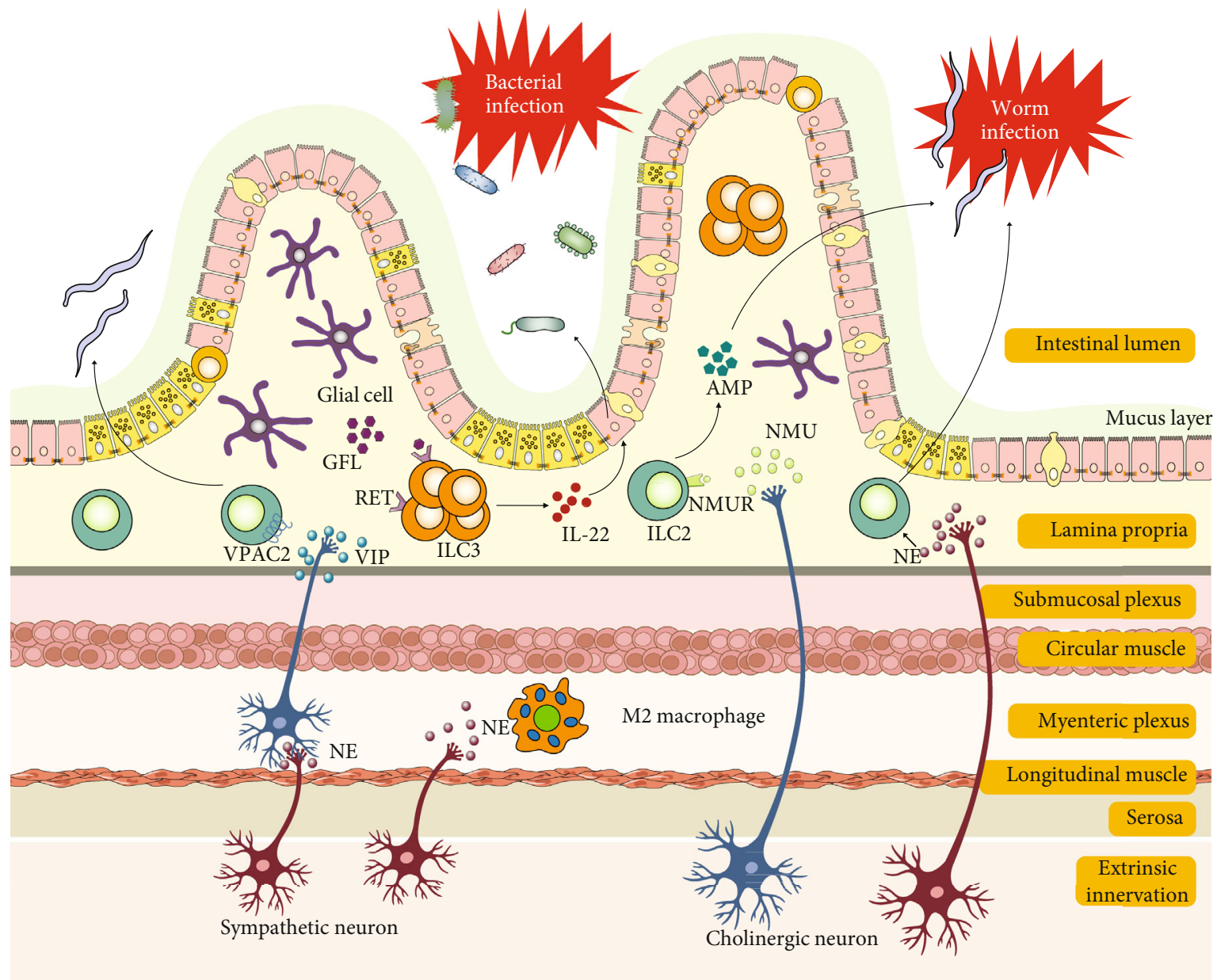

FIGURE 5: Illustration of crosstalk of ILC2 and ILC3 with neurons in helminth infection and gut inflammation, respectively. This image was modified from Reference [54]. Enteric cholinergic neuron-derived NMU activates ILC2 responses and protects against helminth infection. Lamina propria ILC2 function is also regulated by VIP and NE. Glial cell-derived neurotrophic factors stimulate IL-22 production by lamina propria ILC3s, promoting barrier integrity. SNS-derived NE induces a tissue-protective phenotype (M2) in muscularis macrophages. Abbreviations: AMP: antimicrobial peptide; NE: norepinephrine; NMU: neuromedin U; NMUR: neuromedin U receptor; SNS: sympathetic nervous system; GFL: glia cell-derived neurotrophic factor ligand; VIP: vasoactive intestinal peptide; VPAC2: vasoactive intestinal peptide receptor 2 .

3.2. Lipid Mediators. Lipid mediators such as prostaglandin D2 (PGD2) that could regulate ILC2 responses were firstly reported in 2013 [121]. PGD $_{2}$ activates ILC2s from human peripheral blood and increased IL-13 production in the presence of IL-33 and IL-25 [121, 122]. Arachidonic acid metabolite leukotriene D4 (LTD4) was also shown to be able to promote ILC2 activation through the cysteinyl leukotriene receptor 1 (Cys-Lt1R) [123]. Montelukast, a leukotriene receptor antagonist, binds competitively and selectively to Cys-Lt1R. Thus, montelukast may be capable of modulating ILC2 activity. Besides, the arachidonic metabolites lipoxin A4 (LXA4) and macrophage mediator resolving inflammation-1 (maresin-1 or MaR1) can impair the activation of ILC2s $[121,124]$. Therefore, a variety of lipid mediators or inhibitors of these mediators may be developed as ILC modulators [122].

3.3. Cytokines. The cytokines inducing the development and activity of specific subsets of ILCs may also be targeted-such as IL-12 and IL-15 for ILC1s; IL-25, IL-33, and TSLP for ILC2s; and IL-1 $\beta$ and IL-23 for ILC3s, respectively. Interestingly, IL-2, not a classical inducer of ILC activation, was shown to be a critical regulator of ILC2 during pulmonary inflammation [125]. Although the precise involvement of ILCs in specific diseases still remains elusive, treatment blocking these pathways showed some effects in different scenarios besides the intestinal tract. Treatment of multiple sclerosis patients with daclizumab, an antibody targeting IL-2R $\alpha$ (CD25), resulted in an increase in the numbers of NK cells that correlated with drug efficacy [126]. Blockade of CD25 inhibits effector T cell activation, regulatory $\mathrm{T}$ cell expansion and survival, and activation-induced T-cell apoptosis. Because CD25 blockade reduces IL-2 consumption by effector $\mathrm{T}$ cells, it increases IL-2 bioavailability allowing for greater interaction with the intermediate-affinity IL-2R and therefore drives the expansion of CD56 $6^{\text {bright }} \mathrm{NK}$ cells. Unfortunately, daclizumab was withdrawn from the market in 2018 due to severe secondary autoimmune disease 
directed against the central nervous system [127]. In addition, antibodies against IL-25 and IL-33 have shown efficacy in mouse models of allergic lung inflammation [128, 129], and antibody to TSLP intravenously given before allergen challenge in mild asthmatic patients improves asthma symptoms [130].

Apart from cytokines inducing ILC development, effector cytokines such as IFN- $\gamma$, IL-5, and IL-13, or IL-17, produced by ILCs may also be targeted. For example, mepolizumab (antibody to IL-5, NCT01000506) and lebrikizumab (antibody to IL-13, NCT02104674) have been shown effective in clinical trials against asthma $[131,132]$.

3.4. Microbial Compounds. The soluble excretory/secretory products of the helminth parasites impair the activity of ILC2s in response to airway challenges by suppression of IL-33 production [133]. Alternatively, microbial compounds may be used to boost one type of ILC in order to block the other types of ILCs.

\section{Conclusion}

In the past decade, accumulating studies have been carried out to delineate the biology of ILC differentiation, function, and regulation. Yet, still much remains to be investigated. Many discoveries are based on mouse models, and more needs to be described in human scenarios. The prompt response characteristics and antigen-independent activation place ILCs upstream of adaptive response. ILCs possess only few sensory elements for the recognition of nonself, and therefore, ILCs depend on extrinsic cellular sensory elements residing within the tissue [134]. Their crosstalk with T cells, DCs, and other cells need to be deciphered further. ILCs contribute significantly to human health and disease. They play protective roles in some mucosal infections while playing detrimental roles in IBD. Development of modulators to block the detrimental roles of ILCs is of great clinical benefit.

\section{Abbreviations}

$\begin{array}{ll}\text { ILCs: } & \text { Innate lymphoid cells } \\ \text { TJs: } & \text { Tight junctions } \\ \text { MUC2: } & \text { Mucin glycoproteins } \\ \text { TFF: } & \text { Trefoil factor peptides } \\ \text { RELM } \beta \text { : } & \text { Resistin-like molecule } \beta \\ \text { Fcgbp: } & \text { Fc- } \gamma \text { binding protein } \\ \text { TNF- } \alpha: & \text { Tumor necrosis factor- } \alpha \\ \text { LPS: } & \text { Lipopolysaccharide } \\ \text { NF- } \kappa \text { B: } & \text { Nuclear factor- } \kappa \text { B } \\ \text { TLR: } & \text { Toll-like receptor } \\ \text { DCs: } & \text { Dendritic cells } \\ \text { IBD: } & \text { Inflammatory bowel disease } \\ \text { IELs: } & \text { Intraepithelial lymphocytes } \\ \text { IECs: } & \text { Intestinal epithelial cells } \\ \text { TCRs: } & \text { T cell receptors } \\ \text { TSLP: } & \text { Thymic stromal lymphopoietin } \\ \text { S1P: } & \text { Sphingosine } 1 \text {-phosphate } \\ \text { mDCs: } & \text { Migratory dendritic cells } \\ \text { dLNs: } & \text { Draining lymph nodes }\end{array}$

RA: $\quad$ Retinoic acid

PGD2: $\quad$ Prostaglandin D2

NOD2: Nucleotide oligomerization domaincontaining protein 2

RICK: Regulatory protein kinase

Fut2: $\quad$ Fucosyltransferase 2

LTD4: $\quad$ Leukotriene D4

Cys-Lt1R: $\quad$ Cysteinyl leukotriene receptor 1

LXA4: Lipoxin A4

Maresin-1 or MaR1: Macrophage mediator resolving inflammation-1.

\section{Conflicts of Interest}

The authors have declared that no competing interests exist.

\section{Authors' Contributions}

Yuanyuan Wu and Hui Fan wrote the manuscript. Yuanyuan $\mathrm{Wu}$, Aiyun Wang, and Yin Lu constructed the idea. Shijun Wang made valuable comments to the manuscript. Hui Fan and Aiyun Wang contributed equally to this work and share co-first authorship.

\section{Acknowledgments}

This study was supported by grants from the National Natural Science Foundation of China (no. 81703765).

\section{References}

[1] D. Viggiano, G. Ianiro, G. Vanella et al., "Gut barrier in health and disease: focus on childhood," European Review for Medical and Pharmacological Sciences, vol. 19, no. 6, pp. 10771085, 2015.

[2] A. Martin and S. Devkota, "Hold the door: role of the gut barrier in diabetes," Cell Metabolism, vol. 27, no. 5, pp. 949-951, 2018.

[3] D. A. Winer, H. Luck, S. Tsai, and S. Winer, "The intestinal immune system in obesity and insulin resistance," Cell Metabolism, vol. 23, no. 3, pp. 413-426, 2016.

[4] G. J. Randolph, S. Bala, J.-F. Rahier et al., "Lymphoid aggregates remodel lymphatic collecting vessels that serve mesenteric lymph nodes in Crohn disease," The American Journal of Pathology, vol. 186, no. 12, pp. 3066-3073, 2016.

[5] S. C. Bischoff, G. Barbara, W. Buurman et al., "Intestinal permeability - a new target for disease prevention and therapy," BMC Gastroenterology, vol. 14, no. 1, 2014.

[6] M. A. Odenwald and J. R. Turner, "The intestinal epithelial barrier: a therapeutic target?," Nature Reviews Gastroenterology \& Hepatology, vol. 14, no. 1, pp. 9-21, 2017.

[7] C. M. Van Itallie and J. M. Anderson, "Claudins and epithelial paracellular transport," Annual Review of Physiology, vol. 68, no. 1, pp. 403-429, 2005.

[8] C. Chelakkot, J. Ghim, and S. H. Ryu, "Mechanisms regulating intestinal barrier integrity and its pathological implications," Experimental and Molecular Medicine, vol. 50, no. 8, 2018.

[9] S. Zeissig, N. Burgel, D. Gunzel et al., "Changes in expression and distribution of claudin 2, 5 and 8 lead to discontinuous 
tight junctions and barrier dysfunction in active Crohn's disease," Gut, vol. 56, no. 1, pp. 61-72, 2007.

[10] R. Ahmad, R. Chaturvedi, D. Olivares-Villagómez et al., "Targeted colonic claudin-2 expression renders resistance to epithelial injury, induces immune suppression, and protects from colitis," Mucosal Immunology, vol. 7, no. 6, pp. 13401353, 2014.

[11] M. S. Balda, J. A. Whitney, C. Flores, S. González, M. Cereijido, and K. Matter, "Functional dissociation of paracellular permeability and transepithelial electrical resistance and disruption of the apical- basolateral intramembrane diffusion barrier by expression of a mutant tight junction membrane protein," The Journal of Cell Biology, vol. 134, no. 4, pp. 1031-1049, 1996.

[12] M. Saitou, M. Furuse, H. Sasaki et al., "Complex phenotype of mice lacking occludin, a component of tight junction strands," Molecular Biology of the Cell, vol. 11, no. 12, pp. 4131-4142, 2000.

[13] J. D. Schulzke, A. H. Gitter, J. Mankertz et al., "Epithelial transport and barrier function in occludin-deficient mice," Biochimica et Biophysica Acta (BBA) - Biomembranes, vol. 1669, no. 1, pp. 34-42, 2005.

[14] L. Pastorelli, C. SalvoDe, J. R. Mercado, M. Vecchi, and T. T. Pizarro, "Central role of the gut epithelial barrier in the pathogenesis of chronic intestinal inflammation: lessons learned from animal models and human genetics," Frontiers in Immunology, vol. 4, 2013.

[15] L. S. Poritz, K. I. Garver, C. Green, L. Fitzpatrick, F. Ruggiero, and W. A. Koltun, "Loss of the tight junction protein ZO-1 in dextran sulfate sodium induced colitis," The Journal of Surgical Research, vol. 140, no. 1, pp. 12-19, 2007.

[16] H. Schneider, T. Pelaseyed, F. Svensson, and M. E. V. Johansson, "Study of mucin turnover in the small intestine by in vivo labeling," Scientific Reports, vol. 8, no. 1, 2018.

[17] M. Van der Sluis, B. A. E. De Koning, A. C. J. M. De Bruijn et al., "Muc2-deficient mice spontaneously develop colitis, indicating that MUC2 is critical for colonic protection," Gastroenterology, vol. 131, no. 1, pp. 117-129, 2006.

[18] Y. S. Kim and S. B. Ho, "Intestinal goblet cells and mucins in health and disease: recent insights and progress," Current Gastroenterology Reports, vol. 12, no. 5, pp. 319-330, 2010.

[19] R. Bansil and B. S. Turner, "The biology of mucus: composition, synthesis and organization," Advanced Drug Delivery Reviews, vol. 124, pp. 3-15, 2018.

[20] J.-D. Li, W. Feng, M. Gallup et al., “Activation of NF- B via a Src-dependent Ras-MAPK-pp90rsk pathway is required for Pseudomonas aeruginosa-induced mucin overproduction in epithelial cells," Proceedings of the National Academy of Sciences, vol. 95, no. 10, pp. 5718-5723, 2002.

[21] M. Andrianifahanana, N. Moniaux, and S. K. Batra, "Regulation of mucin expression: mechanistic aspects and implications for cancer and inflammatory diseases," Biochimica et Biophysica Acta (BBA) - Reviews on Cancer, vol. 1765, no. 2, pp. 189-222, 2006.

[22] P. Mesquita, N. Jonckheere, R. Almeida et al., "Human MUC2 mucin gene is transcriptionally regulated by Cdx homeodomain proteins in gastrointestinal carcinoma cell lines," The Journal of Biological Chemistry, vol. 278, no. 51, pp. 51549-51556, 2003.

[23] M. van der Sluis, A. Vincent, J. Bouma et al., "Forkhead box transcription factors Foxa1 and Foxa2 are important regula- tors of Muc2 mucin expression in intestinal epithelial cells," Biochemical and Biophysical Research Communications, vol. 369, no. 4, pp. 1108-1113, 2008.

[24] R. Hokari, H. Lee, S. C. Crawley et al., "Vasoactive intestinal peptide upregulates MUC2 intestinal mucin via CREB/ATF1," American Journal of Physiology-Gastrointestinal and Liver Physiology, vol. 289, no. 5, pp. G949-G959, 2005.

[25] N. Yamada, T. Hamada, M. Goto et al., "MUC2 expression is regulated by histone $\mathrm{H} 3$ modification and DNA methylation in pancreatic cancer," International Journal of Cancer, vol. 119, no. 8, pp. 1850-1857, 2006.

[26] K. Okudaira, S. Kakar, L. Cun et al., "MUC2 gene promoter methylation in mucinous and non-mucinous colorectal cancer tissues," International Journal of Oncology, vol. 36, no. 4, pp. 765-775, 2010.

[27] T. Sato, J. H. van Es, H. J. Snippert et al., "Paneth cells constitute the niche for Lgr5 stem cells in intestinal crypts," Nature, vol. 469, no. 7330, pp. 415-418, 2011.

[28] S. Vaishnava, C. L. Behrendt, A. S. Ismail, L. Eckmann, and L. V. Hooper, "Paneth cells directly sense gut commensals and maintain homeostasis at the intestinal host-microbial interface," Proceedings of the National Academy of Sciences, vol. 105, no. 52, pp. 20858-20863, 2008.

[29] M. Schmitt, M. Schewe, A. Sacchetti et al., "Paneth cells respond to inflammation and contribute to tissue regeneration by acquiring stem-like features through SCF/c-Kit signaling," Cell Reports, vol. 24, no. 9, pp. 2312-2328.e7, 2018.

[30] M. M. Wouters, M. Vicario, and J. Santos, "The role of mast cells in functional GI disorders," Gut, vol. 65, no. 1, pp. 155-168, 2016.

[31] E. A. Mayer, K. Tillisch, and A. Gupta, "Gut/brain axis and the microbiota," Journal of Clinical Investigation, vol. 125, no. 3, pp. 926-938, 2015.

[32] S. Buhner and M. Schemann, "Mast cell-nerve axis with a focus on the human gut," Biochimica et Biophysica Acta Molecular Basis of Disease, vol. 1822, no. 1, pp. 85-92, 2012.

[33] L. L. Reber, R. Sibilano, K. Mukai, and S. J. Galli, "Potential effector and immunoregulatory functions of mast cells in mucosal immunity," Mucosal Immunology, vol. 8, no. 3, pp. 444-463, 2015.

[34] G. Barbara, V. Stanghellini, R. De Giorgio et al., "Activated mast cells in proximity to colonic nerves correlate with abdominal pain in irritable bowel syndrome," Gastroenterology, vol. 126, no. 3, pp. 693-702, 2004.

[35] K. R. Groschwitz, R. Ahrens, H. Osterfeld et al., "Mast cells regulate homeostatic intestinal epithelial migration and barrier function by a chymase/Mcpt4-dependent mechanism," Proceedings of the National Academy of Sciences, vol. 106, no. 52, pp. 22381-22386, 2009.

[36] S. J. Galli, M. Grimbaldeston, and M. Tsai, "Immunomodulatory mast cells: negative, as well as positive, regulators of immunity," Nature Reviews Immunology, vol. 8, no. 6, pp. 478-486, 2008.

[37] E. Z. M. da Silva, M. C. Jamur, and C. Oliver, "Mast cell function: a new vision of an old cell," Journal of Histochemistry and Cytochemistry, vol. 62, no. 10, pp. 698-738, 2014.

[38] S. Y. Chang, H. J. Ko, and M. N. Kweon, "Mucosal dendritic cells shape mucosal immunity," Experimental and Molecular Medicine, vol. 46, no. 3, pp. e84-e84, 2014.

[39] M. Rimoldi, M. Chieppa, M. Vulcano, P. Allavena, and M. Rescigno, "Intestinal epithelial cells control dendritic cell 
function," Annals of the New York Academy of Sciences, vol. 1029, no. 1, pp. 66-74, 2004.

[40] M. Becker, S. Güttler, A. Bachem et al., "Ontogenic, phenotypic, and functional characterization of XCR1+ dendritic cells leads to a consistent classification of intestinal dendritic cells based on the expression of XCR 1 and SIRP $\alpha$," Frontiers in Immunology, vol. 5, 2014.

[41] J. R. McDole, L. W. Wheeler, K. G. McDonald et al., "Goblet cells deliver luminal antigen to CD103+ dendritic cells in the small intestine," Nature, vol. 483, no. 7389, pp. 345-349, 2012.

[42] J. Farache, I. Koren, I. Milo et al., "Luminal bacteria recruit CD103+ dendritic cells into the intestinal epithelium to sample bacterial antigens for presentation," Immunity, vol. 38, no. 3, pp. 581-595, 2013.

[43] M. Gross, T. M. Salame, and S. Jung, "Guardians of the gut murine intestinal macrophages and dendritic cells," Frontiers in Immunology, vol. 6, 2015.

[44] C. C. Bain and A. Schridde, "Origin, differentiation, and function of intestinal macrophages," Frontiers in Immunology, vol. 9, 2018.

[45] J. R. Grainger, J. E. Konkel, T. Zangerle-Murray, and T. N. Shaw, "Macrophages in gastrointestinal homeostasis and inflammation," Pflügers Archiv - European Journal of Physiology, vol. 469, no. 3-4, pp. 527-539, 2017.

[46] E. Zigmond, B. Bernshtein, G. Friedlander et al., "Macrophage-restricted interleukin-10 receptor deficiency, but not IL-10 deficiency, causes severe spontaneous colitis," Immunity, vol. 40, no. 5, pp. 720-733, 2014.

[47] L. Deng, J.-F. Zhou, R. S. Sellers et al., "A novel mouse model of inflammatory bowel disease links mammalian target of rapamycin-dependent hyperproliferation of colonic epithelium to inflammation-associated tumorigenesis," The American Journal of Pathology, vol. 176, no. 2, pp. 952967, 2010.

[48] C. C. Bain, A. Bravo-Blas, C. L. Scott et al., "Constant replenishment from circulating monocytes maintains the macrophage pool in the intestine of adult mice," Nature Immunology, vol. 15, no. 10, pp. 929-937, 2014.

[49] C. C. Bain, C. L. Scott, H. Uronen-Hansson et al., "Resident and pro-inflammatory macrophages in the colon represent alternative context-dependent fates of the same Ly6C hi monocyte precursors," Mucosal Immunology, vol. 6, no. 3, pp. 498-510, 2013.

[50] S. De Schepper, S. Verheijden, J. Aguilera-Lizarraga et al., "Self-maintaining gut macrophages are essential for intestinal homeostasis," Cell, vol. 175, no. 2, pp. 400-415.e13, 2018.

[51] H. Cheroutre, F. Lambolez, and D. Mucida, "The light and dark sides of intestinal intraepithelial lymphocytes," Nature Reviews Immunology, vol. 11, no. 7, pp. 445-456, 2011.

[52] D. Olivares-Villagómez and L. Van Kaer, "Intestinal intraepithelial lymphocytes: sentinels of the mucosal barrier," Trends in Immunology, vol. 39, no. 4, pp. 264-275, 2018.

[53] D. Masopust, D. Choo, V. Vezys et al., "Dynamic T cell migration program provides resident memory within intestinal epithelium," The Journal of Experimental Medicine, vol. 207, no. 3, pp. 553-564, 2010.

[54] C. Godinho-Silva, F. Cardoso, and H. Veiga-Fernandes, "Neuro-immune cell units: a new paradigm in physiology," Annual Review of Immunology, vol. 37, no. 1, pp. 19-46, 2019.
[55] M. D. Gershon, "The enteric nervous system: a second brain," Hospital Practice, vol. 34, no. 7, pp. 31-52, 1999.

[56] J. B. Furness, "Types of neurons in the enteric nervous system," Journal of the Autonomic Nervous System, vol. 81, no. 1-3, pp. 87-96, 2000.

[57] G. Matteoli, P. J. Gomez-Pinilla, A. Nemethova et al., "A distinct vagal anti-inflammatory pathway modulates intestinal muscularis resident macrophages independent of the spleen," Gut, vol. 63, no. 6, pp. 938-948, 2014.

[58] T. G. Bush, T. C. Savidge, T. C. Freeman, H. J. Cox, and E. A. Campbell, "Fulminant jejuno-ileitis induced by ablation of enteric glia in adult transgenic mice," Gastroenterology, vol. 120, no. 5, pp. A186-A187, 2001.

[59] A. Cornet, T. C. Savidge, J. Cabarrocas et al., "Enterocolitis induced by autoimmune targeting of enteric glial cells: a possible mechanism in Crohn's disease?," Proceedings of the National Academy of Sciences of the United States of America, vol. 98, no. 23, pp. 13306-13311, 2001.

[60] M. Rao, D. Rastelli, L. Dong et al., "Enteric glia regulate gastrointestinal motility but are not required for maintenance of the epithelium in mice," Gastroenterology, vol. 153, no. 4, pp. 1068-1081.e7, 2017.

[61] E. Vivier, D. Artis, M. Colonna et al., "Innate lymphoid cells: 10 years on," Cell, vol. 174, pp. 1054-1066, 2018.

[62] M. E. Kotas and R. M. Locksley, "Why innate lymphoid cells?," Immunity, vol. 48, pp. 1081-1090, 2018.

[63] E. Klein and H. Wigzell, "“Natural” killer cells in the mouse. I. Cytotoxic cells with specificity for mouse Moloney leukemia cells. Specificity and distribution according to genotype," vol. 5, no. 2, pp. 112-117, 1975.

[64] Y.-J. Park, D.-S. Kuen, and Y. Chung, "Future prospects of immune checkpoint blockade in cancer: from response prediction to overcoming resistance," Experimental \& Molecular Medicine, vol. 50, p. 109, 2018.

[65] C. A. J. Vosshenrich, M. E. García-Ojeda, S. I. SamsonVilléger et al., "A thymic pathway of mouse natural killer cell development characterized by expression of GATA-3 and CD127," Nature Immunology, vol. 7, no. 11, pp. 1217-1224, 2006.

[66] M. Cella, A. Fuchs, W. Vermi et al., "A human natural killer cell subset provides an innate source of IL-22 for mucosal immunity," Nature, vol. 457, no. 7230 , pp. $722-$ 725, 2009

[67] A. E. Price, H.-E. Liang, B. M. Sullivan et al., "Systemically dispersed innate IL-13-expressing cells in type 2 immunity," Proceedings of the National Academy of Sciences of the United States of America, vol. 107, no. 25, pp. 11489-11494, 2010.

[68] S. Buonocore, P. P. Ahern, H. H. Uhlig et al., "Innate lymphoid cells drive interleukin-23-dependent innate intestinal pathology," Nature, vol. 464, no. 7293, pp. 1371-1375, 2010.

[69] L. A. Monticelli, G. F. Sonnenberg, M. C. Abt et al., "Innate lymphoid cells promote lung-tissue homeostasis after infection with influenza virus," Nature Immunology, vol. 12, no. 11, pp. 1045-1054, 2011.

[70] J. H. Bernink, C. P. Peters, M. Munneke et al., "Human type 1 innate lymphoid cells accumulate in inflamed mucosal tissues," Nature Immunology, vol. 14, no. 3, pp. 221-229, 2013.

[71] A. Fuchs, W. Vermi, J. S. Lee et al., "Intraepithelial type 1 innate lymphoid cells are a unique subset of IL-12- and IL15-responsive IFN- $\gamma$-producing cells," Immunity, vol. 38, no. 4, pp. 769-781, 2013. 
[72] M. R. Hepworth, L. A. Monticelli, T. C. Fung et al., "Innate lymphoid cells regulate CD4 + T-cell responses to intestinal commensal bacteria," Nature, vol. 498, no. 7452, pp. 113117, 2013.

[73] D. Artis and H. Spits, "The biology of innate lymphoid cells," Nature, vol. 517, no. 7534, pp. 293-301, 2015.

[74] S. Middendorp and E. E. S. Nieuwenhuis, "NKT cells in mucosal immunity," Mucosal Immunology, vol. 2, no. 5, pp. 393-402, 2009.

[75] C. Ma, M. Han, B. Heinrich et al., "Gut microbiome-mediated bile acid metabolism regulates liver cancer via NKT cells," Science, vol. 360, 2018.

[76] L. Han, X.-m. Wang, S. Di et al., "Innate lymphoid cells: a link between the nervous system and microbiota in intestinal networks," Mediators of Inflammation, vol. 2019, Article ID 1978094, 11 pages, 2019.

[77] M. Bruchard and F. Ghiringhelli, "Deciphering the roles of innate lymphoid cells in cancer," Frontiers in Immunology, vol. 10, 2019.

[78] C. J. Oliphant, Y. Y. Hwang, J. A. Walker et al., "MHCIImediated dialog between group 2 innate lymphoid cells and CD $4+$ T cells potentiates type 2 immunity and promotes parasitic helminth expulsion," Immunity, vol. 41, no. 2, pp. 283295, 2014.

[79] M. Wagner, K. Moro, and S. Koyasu, "Plastic heterogeneity of innate lymphoid cells in cancer," Trends in Cancer, vol. 3, no. 5, pp. 326-335, 2017.

[80] Y. Huang, K. Mao, X. Chen et al., "S1P-dependent interorgan trafficking of group 2 innate lymphoid cells supports host defense," Science, vol. 359, no. 6371, pp. 114-119, 2018.

[81] M. Colonna, "Innate lymphoid cells: diversity, plasticity, and unique functions in immunity," Immunity, vol. 48, pp. 11041117, 2018.

[82] A. I. Lim, Y. Li, S. Lopez-Lastra et al., "Systemic human ILC precursors provide a substrate for tissue ILC differentiation," Cell, vol. 168, no. 6, pp. 1086-1100.e10, 2017.

[83] J. H. Bernink, L. Krabbendam, K. Germar et al., "Interleukin12 and -23 control plasticity of Cd127+group 1 and group 3 innate lymphoid cells in the intestinal lamina propria," Immunity, vol. 43, no. 1, pp. 146-160, 2015.

[84] O.-E. Weizman, N. M. Adams, I. S. Schuster et al., "ILC1 confer early host protection at initial sites of viral infection," Cell, vol. 171, no. 4, pp. 795-808.e12, 2017.

[85] E. Wong, R.-H. Xu, D. Rubio et al., "Migratory dendritic cells, group 1 innate lymphoid cells, and inflammatory monocytes collaborate to recruit NK cells to the virusinfected lymph node," Cell Reports, vol. 24, no. 1, pp. 142-154, 2018.

[86] T. Y. F. Halim, Y. Y. Hwang, S. T. Scanlon et al., "Group 2 innate lymphoid cells license dendritic cells to potentiate memory TH2 cell responses," Nature Immunology, vol. 17, no. 1, pp. 57-64, 2016.

[87] T. Y. F. Halim, C. A. Steer, L. Mathä et al., "Group 2 innate lymphoid cells are critical for the initiation of adaptive $\mathrm{T}$ helper 2 cell-mediated allergic lung inflammation," Immunity, vol. 40, no. 3, pp. 425-435, 2014.

[88] F. Flores-Borja, S. Irshad, P. Gordon et al., "Crosstalk between innate lymphoid cells and other immune cells in the tumor microenvironment," Journal of Immunology Research, vol. 2016, Article ID 7803091, 14 pages, 2016.
[89] A. Rivera, M. C. Siracusa, G. S. Yap, and W. C. Gause, "Innate cell communication kick-starts pathogen-specific immunity," Nature Immunology, vol. 17, no. 4, pp. 356-363, 2016.

[90] C. S. N. Klose, M. Flach, L. Möhle et al., "Differentiation of type 1 ILCs from a common progenitor to all helper-like innate lymphoid cell lineages," Cell, vol. 157, no. 2, pp. 340356, 2014.

[91] S. Boulenouar, X. Michelet, D. Duquette et al., "Adipose type one innate lymphoid cells regulate macrophage homeostasis through targeted cytotoxicity," Immunity, vol. 46, no. 2, pp. 273-286, 2017.

[92] T. E. O’Sullivan, M. Rapp, X. Fan et al., “Adipose-resident group 1 innate lymphoid cells promote obesity-associated insulin resistance," Immunity, vol. 45, no. 2, pp. 428-441, 2016.

[93] S. J. Van Dyken and R. M. Locksley, "Interleukin-4- and interleukin-13-mediated alternatively activated macrophages: roles in homeostasis and disease," Annual Review of Immunology, vol. 31, no. 1, pp. 317-343, 2013.

[94] A.-G. Besnard, R. Guabiraba, W. Niedbala et al., "IL-33mediated protection against experimental cerebral malaria is linked to induction of type 2 innate lymphoid cells, M2 macrophages and regulatory T cells," PLoS Pathogens, vol. 11, no. 2, 2015.

[95] M.-W. Lee, J. I. Odegaard, L. Mukundan et al., "Activated type 2 innate lymphoid cells regulate beige fat biogenesis," Cell, vol. 160, no. 1-2, pp. 74-87, 2015.

[96] A. B. Molofsky, J. C. Nussbaum, H.-E. Liang et al., "Innate lymphoid type 2 cells sustain visceral adipose tissue eosinophils and alternatively activated macrophages," The Journal of Experimental Medicine, vol. 210, no. 3, pp. 535-549, 2013.

[97] G. Gasteiger and A. Y. Rudensky, "Interactions between innate and adaptive lymphocytes," Nature Reviews Immunology, vol. 14, no. 9, pp. 631-639, 2014.

[98] A. Mortha, A. Chudnovskiy, D. Hashimoto et al., "Microbiota-dependent crosstalk between macrophages and ILC3 promotes intestinal homeostasis," Science, vol. 343, no. 6178, pp. 1249288-1249288, 2014.

[99] A. Geremia and C. V. Arancibia-Cárcamo, "Innate lymphoid cells in intestinal inflammation," Frontiers in Immunology, vol. 8, 2017.

[100] N. Powell, A. W. Walker, E. Stolarczyk et al., “The transcription factor T-bet regulates intestinal inflammation mediated by interleukin-7 receptor+ innate lymphoid cells," Immunity, vol. 37, no. 4, pp. 674-684, 2012.

[101] C. S. N. Klose, E. A. Kiss, V. Schwierzeck et al., "A T-bet gradient controls the fate and function of CCR6-ROR $\gamma \mathrm{t}+$ innate lymphoid cells," Nature, vol. 494, no. 7436, pp. 261-265, 2013.

[102] E. D. T. Wojno, L. A. Monticelli, S. V. Tran et al., "The prostaglandin D2 receptor CRTH2 regulates accumulation of group 2 innate lymphoid cells in the inflamed lung," Mucosal Immunology, vol. 8, no. 6, pp. 1313-1323, 2015.

[103] T. A. Doherty, N. Khorram, S. Lund, A. K. Mehta, M. Croft, and D. H. Broide, "Lung type 2 innate lymphoid cells express cysteinyl leukotriene receptor 1 , which regulates $\mathrm{TH} 2$ cytokine production," The Journal of Allergy and Clinical Immunology, vol. 132, no. 1, pp. 205-213, 2013.

[104] B. Zeng, S. Shi, G. Ashworth, C. Dong, J. Liu, and F. Xing, "ILC3 function as a double-edged sword in inflammatory bowel diseases," Cell Death \& Disease, vol. 10, no. 4, 2019. 
[105] N. Barnich, J. E. Aguirre, H. C. Reinecker, R. Xavier, and D. K. Podolsky, "Membrane recruitment of NOD2 in intestinal epithelial cells is essential for nuclear factor $-\kappa \mathrm{B}$ activation in muramyl dipeptide recognition," The Journal of Cell Biology, vol. 170, no. 1, pp. 21-26, 2005.

[106] C. A. Lindemans, M. Calafiore, A. M. Mertelsmann et al., "Interleukin-22 promotes intestinal-stem-cell-mediated epithelial regeneration," Nature, vol. 528, no. 7583, pp. 560$564,2015$.

[107] J. A. Dudakov, A. M. Hanash, and M. R. M. van den Brink, "Interleukin-22: immunobiology and pathology," Annual Review of Immunology, vol. 33, no. 1, pp. 747-785, 2015.

[108] Y. Goto, T. Obata, J. Kunisawa et al., "Innate lymphoid cells regulate intestinal epithelial cell glycosylation," Science, vol. 345, no. 6202, pp. 1254009-1254009, 2014.

[109] C. Pearson, H. H. Uhlig, and F. Powrie, "Lymphoid microenvironments and innate lymphoid cells in the gut," Trends in Immunology, vol. 33, no. 6, pp. 289-296, 2012.

[110] H. Veiga-Fernandes and D. Artis, "Neuronal-immune system cross-talk in homeostasis," Science, vol. 359, no. 6383, pp. 1465-1466, 2018.

[111] C. S. N. Klose, T. Mahlakõiv, J. B. Moeller et al., "The neuropeptide neuromedin $U$ stimulates innate lymphoid cells and type 2 inflammation," Nature, vol. 549, no. 7671, pp. 282286, 2017.

[112] V. Cardoso, J. Chesné, H. Ribeiro et al., "Neuronal regulation of type 2 innate lymphoid cells via neuromedin U," Nature, vol. 549, no. 7671, pp. 277-281, 2017.

[113] S. Moriyama, J. R. Brestoff, A.-L. Flamar et al., “ $\beta 2$-Adrenergic receptor-mediated negative regulation of group 2 innate lymphoid cell responses," Science, vol. 359, no. 6379, pp. 1056-1061, 2018.

[114] S. Ibiza, B. García-Cassani, H. Ribeiro et al., "Glial-cellderived neuroregulators control type 3 innate lymphoid cells and gut defence," Nature, vol. 535, pp. 440-443, 2016.

[115] J. R. Huh, M. W. L. Leung, P. Huang et al., "Digoxin and its derivatives suppress T H17 cell differentiation by antagonizing ROR 3 3t activity," Nature, vol. 472, no. 7344, pp. 486490, 2011.

[116] U. Guendisch, J. Weiss, F. Ecoeur et al., "Pharmacological inhibition of ROR $\gamma \mathrm{t}$ suppresses the Th17 pathway and alleviates arthritis in vivo," PLoS One, vol. 12, no. 11, 2017.

[117] I. Dulubova, X. Jiang, I. Trevino et al., "RTA 1701 is an orallybioavailable, potent, and selective ROR $\gamma$ t inhibitor that suppresses Th17 differentiation in vitro and is efficacious in mouse models of autoimmune disease," Journal of Immunology, vol. 200, 2018.

[118] K. Venken, P. Jacques, C. Mortier et al., "ROR $\gamma$ t inhibition selectively targets IL-17 producing iNKT and $\gamma \delta$-T cells enriched in spondyloarthritis patients," Nature Communications, vol. 10, no. 1, 2019.

[119] G. Eberl, M. Colonna, J. P. D. Santo, and A. N. J. McKenzie, "Innate lymphoid cells: a new paradigm in immunology," Science, vol. 348, no. 6237, pp. aaa6566-aaa6566, 2015.

[120] N. Garrido-Mesa, J.-H. Schroeder, E. Stolarczyk et al., "T-bet controls intestinal mucosa immune responses via repression of type 2 innate lymphoid cell function," Mucosal Immunology, vol. 12, no. 1, pp. 51-63, 2019.

[121] C. Barnig, M. Cernadas, S. Dutile et al., "Lipoxin A4 regulates natural killer cell and type 2 innate lymphoid cell activation in asthma," Science Translational Medicine, vol. 5, no. 174, pp. 174ra26-174ra26, 2013.

[122] T. A. Doherty and D. H. Broide, "Lipid regulation of group 2 innate lymphoid cell function: moving beyond epithelial cytokines," The Journal of Allergy and Clinical Immunology, vol. 141, no. 5, pp. 1587-1589, 2018.

[123] L. Xue, M. Salimi, I. Panse et al., "Prostaglandin D 2 activates group 2 innate lymphoid cells through chemoattractant receptor-homologous molecule expressed on T H 2 cells," The Journal of Allergy and Clinical Immunology, vol. 133, no. 4, pp. 1184-1194.e7, 2014.

[124] N. Krishnamoorthy, P. R. Burkett, J. Dalli et al., "Cutting edge: maresin-1 engages regulatory $\mathrm{T}$ cells to limit type 2 innate lymphoid cell activation and promote resolution of lung inflammation," Journal of Immunology, vol. 194, no. 3, pp. 863-867, 2015.

[125] B. Roediger, R. Kyle, S. S. Tay et al., "IL-2 is a critical regulator of group 2 innate lymphoid cell function during pulmonary inflammation," The Journal of Allergy and Clinical Immunology, vol. 136, no. 6, pp. 1653-1663.e7, 2015.

[126] J. S. A. Perry, S. Han, Q. Xu et al., "Inhibition of LTi cell development by CD25 blockade is associated with decreased intrathecal inflammation in multiple sclerosis," Science Translational Medicine, vol. 4, no. 145, pp. 145ra106-145ra106, 2012.

[127] S. L. Cohan, E. B. Lucassen, M. C. Romba, and S. N. Linch, "Daclizumab: mechanisms of action, therapeutic efficacy, adverse events and its uncovering the potential role of innate immune system recruitment as a treatment strategy for relapsing multiple sclerosis," Biomedicine, vol. 7, no. 1, 2019.

[128] S. J. Ballantyne, J. L. Barlow, H. E. Jolin et al., "Blocking IL-25 prevents airway hyperresponsiveness in allergic asthma," The Journal of Allergy and Clinical Immunology, vol. 120, no. 6, pp. 1324-1331, 2007.

[129] A. J. Coyle, C. Lloyd, J. Tian et al., "Crucial role of the interleukin 1 receptor family member T1/St2 in T helper cell type 2-mediated lung mucosal immune responses," The Journal of Experimental Medicine, vol. 190, no. 7, pp. 895-902, 2002.

[130] G. M. Gauvreau, P. M. O'Byrne, L.-P. Boulet et al., "Effects of an anti-TSLP antibody on allergen-induced asthmatic responses," The New England Journal of Medicine, vol. 370, no. 22, pp. 2102-2110, 2014.

[131] I. D. Pavord, S. Korn, P. Howarth et al., "Mepolizumab for severe eosinophilic asthma (DREAM): a multicentre, double-blind, placebo-controlled trial," Lancet, vol. 380, no. 9842, pp. 651-659, 2012.

[132] P. Korenblat, E. Kerwin, I. Leshchenko et al., "Efficacy and safety of lebrikizumab in adult patients with mild-tomoderate asthma not receiving inhaled corticosteroids," Respiratory Medicine, vol. 134, pp. 143-149, 2018.

[133] H. J. McSorley, N. F. Blair, K. A. Smith, A. N. J. McKenzie, and R. M. Maizels, "Blockade of IL-33 release and suppression of type 2 innate lymphoid cell responses by helminth secreted products in airway allergy," Mucosal Immunology, vol. 7, no. 5, pp. 1068-1078, 2014.

[134] A. Mortha and K. Burrows, "Cytokine networks between innate lymphoid cells and myeloid cells," Frontiers in Immunology, vol. 9, 2018. 


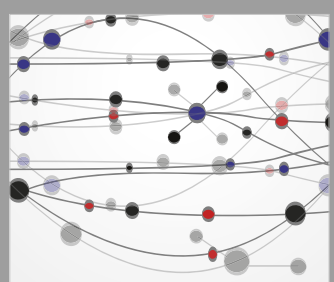

The Scientific World Journal
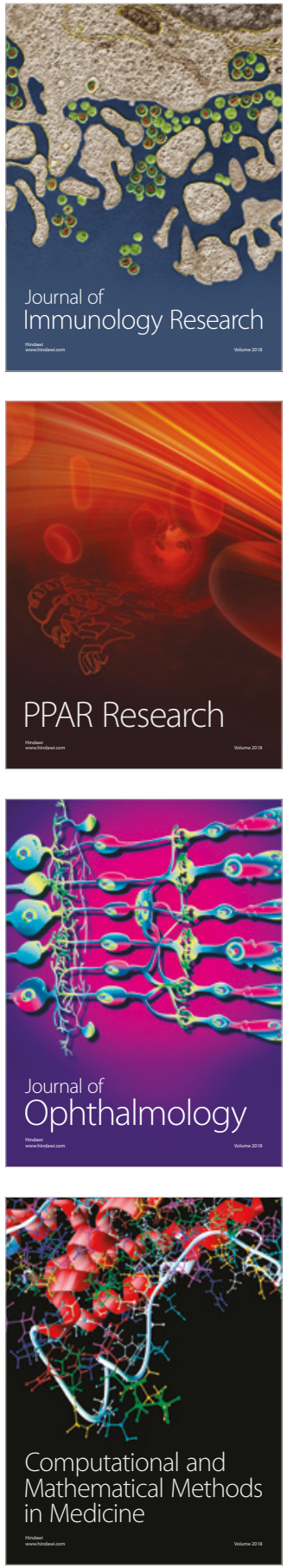

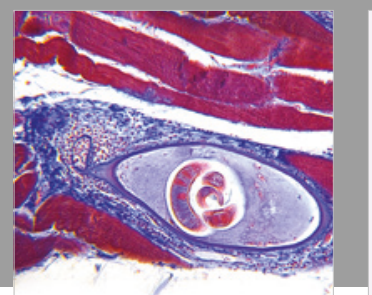

Gastroenterology Research and Practice

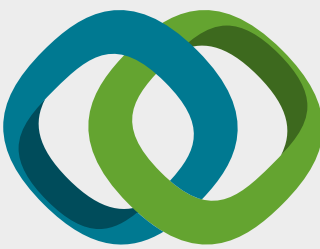

\section{Hindawi}

Submit your manuscripts at

www.hindawi.com
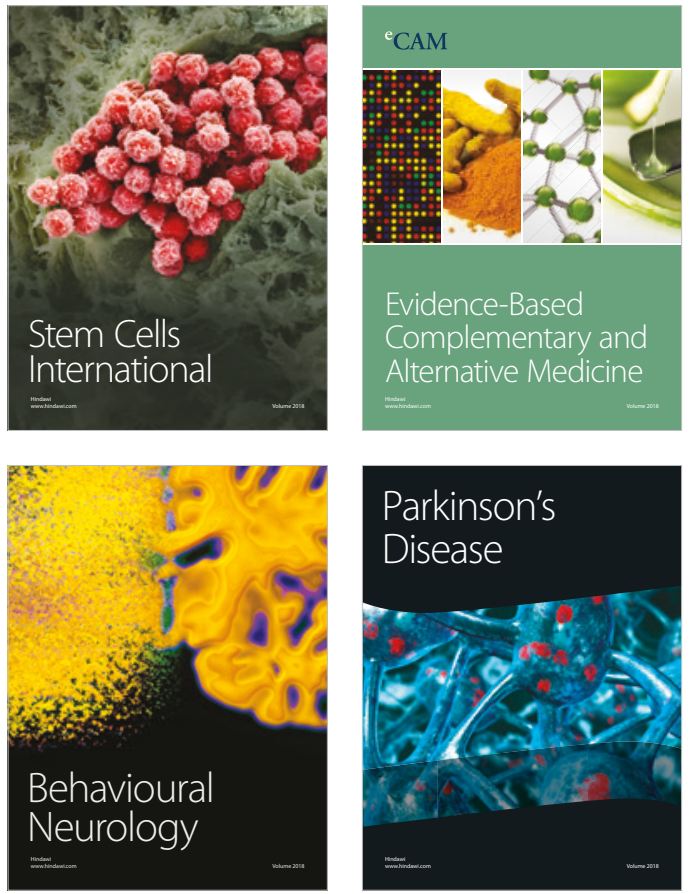

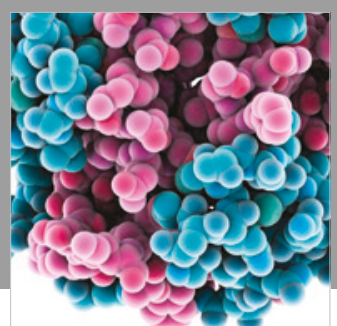

ournal of

Diabetes Research

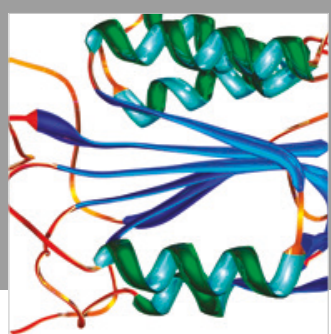

Disease Markers
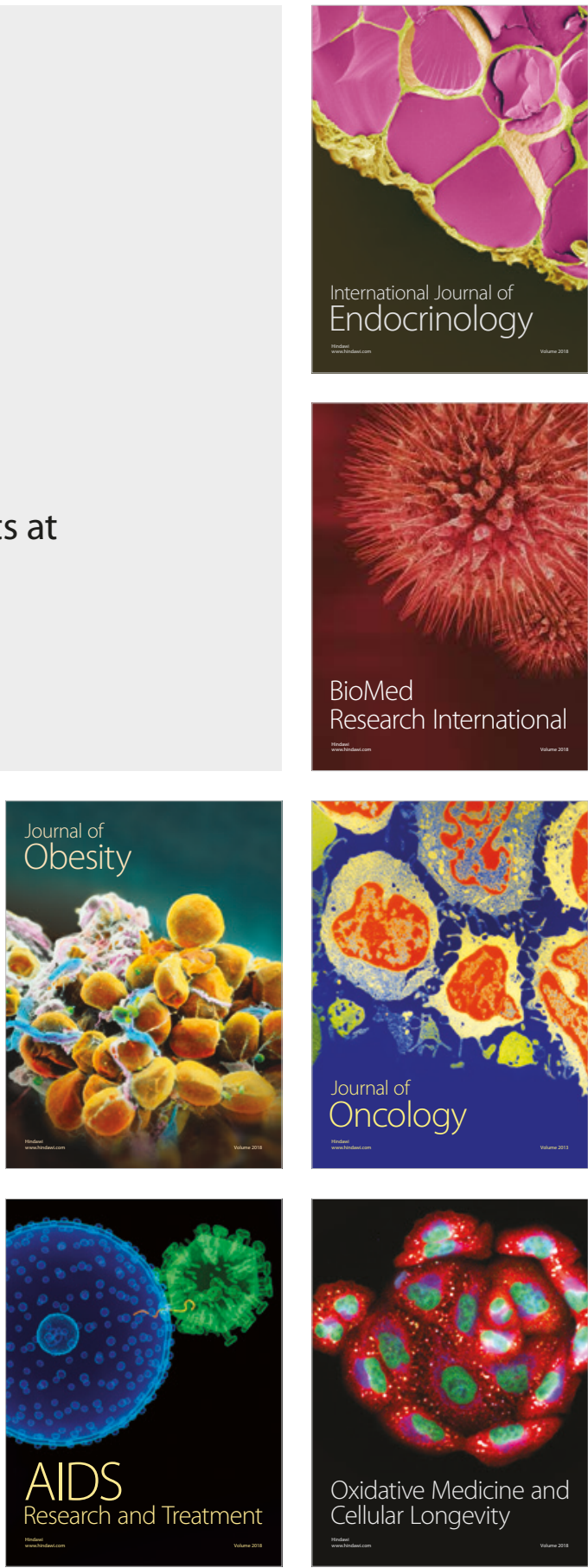